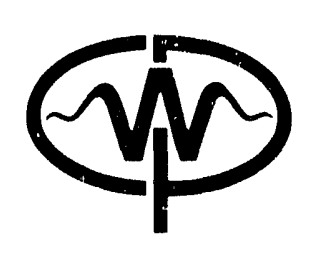

\title{
Compensating finite-difference errors in 3-D migration and modeling
}

\author{
by
}

Zhiming Li

Center for Wave Phenomena

Colorado School of Mines

Golden, Colorado 8(0.4()1

Phone (3)3) 27.3-3.5.57 


\title{
Compensating finite-difference errors in 3-D migration and modeling
}

\author{
Zhiming Li
}

\begin{abstract}
One-pass three-dimensional (3-D) depth migration potentially offers more accurate imaging results than does conventional two-pass $3-\mathrm{D}$ migration, in variable velocity media. Conventional one-pass $3-D$ migration, done with the method of finite-difference inline and crossline splitting, however, creates large errors in imaging complex structures due to paraxial wave-equation approximation of the one-way wave equation, inline-crossline splitting, and finite-difference grid dispersion.

After analyzing the finite-difference errors in conventional 3-D poststack wave field extrapolation, the paper presents a method that compensates for the crrors and yet still preserves the efficiency of the conventional finite-difference splitting method. For frequency-space 3-D finite-difference migration and modeling, the compensation uperator is implemented using the phase-shift method, or phaseshift plus interpolation method, depending on the extent of lateral velocity variations. The compensation operator increases the accuracy of handling steep dips, suppresses the inline and crossline splitting error, and reduces finite-difference grid dispersions. Numerical calculations show that the quality of 3-D migration and 3-D modeling is improved significantly with the finite-difference error compensation method presented in this paper.
\end{abstract}

\section{INTRODUCTION}

Single-pass, as opposed to two-pass, 3-D wave-equation migration has been arvocated for imaging of common-midpoint (CMP) stacked 3-D seismic data for some years, primarily where velocity varies both vertically and laterally (Yilmaz, 1987). Finite-difference implementations of one-pass 3-D migration often use the inline $(x)$ and crossline $(y)$ splitting technique in each step of wave field extrapolation (Brown, 1983). While the splitting technique affords computational efficiency, known errons of 
positioning steeply dipping reflectors result, especially when the $x$ - and $y$ - directions are away from the dominant dip direction of the area.

Many approaches had been taken in the past 10 years to overcome the problem of mispositioning stecp dips due to the $x-y$ splitting in 3-D migration. Ristow (1980) suggested further splitting along the two diagonal directions $(x= \pm y)$, besides splitting along $x$ and $y$ in each downward extrapolation step. Kitchenside (1988) used the method of phase-shift migration plus finite-difference residual wave-field extrapolation to reduce the error due to splitting. Liraves is.d Clayton (1990) proposed implementing a phase-correction operator using finite-differences with damping function (to ensure stability) in their 3 -D paraxial wave-equation modeling of seismic wave field. Hale (1990) proposed a 3-D, explicit finite-difference migration using McClellan transforrations, as an alternative to $x-y$ splitting.

Instead of using phase-shift migration plus finite-difference residual wave-ficld extrapolation in Kitchenside's approach, I use the conventional finite-difference migration plus phase-shift residual wave-field extrapolation to improve the accuracy of 3-D finite-difference migration. Without any changes to the existing conventional one-pass 3-D implicit finite-difference migration in the migration part, I simply add the error compensation as a phase-shift filter at certain steps of downward extrapolation. The method presented in this paper compensates not only for the splitting error, but also for steep-dip positioning error and finite-difference dispersion error, by using Gazdag's (1978) method of phase shift, where, instead of using the wave equation, I use what I shall call the finite-difference-error compensation equation. In the presence of strong lateral velocity variations, again, the method of Gazdag's phase shift plus interpolation (Gazdag and Sguazzero, 1984) is used to implement the finite-difference-error compensation equation.

\section{PARAXIAL EQUATIONS and INLINE-CROSSLINE SPLITTING}

The 3-D acoustic wave equation for upcoming waves in the frequency-space domain $(\omega, x, y, z)$ can be written as,

$$
\frac{\partial P}{\partial z}=\frac{i \omega}{v(x, y, z)} \sqrt{1+\frac{v^{2}(x, y, z)}{\omega^{2}}\left(\frac{\partial^{2}}{\partial x^{2}}+\frac{\partial^{2}}{\partial y^{2}}\right)} P,
$$

where $P=P(\omega, x, y, z)$ is the wave field, $\omega$ is radial frequency, $x$ is the lateral cuordinate along the inline direction, $y$ is the lateral coordinate along the crossline dire tion, $z$ is depth, and $v(x, y, z)$ is velocity.

To solve equation (1) in the $(\omega, x, y, z)$ domain numerically, the square-root operator must be expanded and approximated with a certain order of paraxial equation, depending on the accuracy of approximation. Using the continuous fractional expansion (Claerbout, 1985) of the square root operator and facturization of the expansion (Ma, 1982), equation (1) can be approximated with the following paraxial equation of order $2 n$, 


$$
\frac{\partial P}{\partial z}=\frac{i \omega}{v(x, y, z)}\left[1+\sum_{i=1}^{n} \frac{\alpha_{i} S}{1+\beta_{i} S}\right] P
$$

where $\alpha_{i}$ and $\beta_{i}$ are expansion coefficients given by Lee and Sull (1985), $S=S_{x}+S_{y}$ and $S_{x}=\left(v^{2}(x, y, z) / \omega^{2}\right) \partial^{2} / \partial x^{2}, S_{y}=\left(v^{2}(x, y, z) / \omega^{2}\right) \partial^{2} / \partial y^{2}$.

The higher the order $2 n$, the better equation (2) approximates equation (1) in handling steep dips. In practice, the paraxial equation with $n=1$ yields good accuracy for dips up to 65 degrees (Yilmaz, 1987). Equation (2) can be solved using a splitting method, resulting in the following sequence of $(n+1)$ equations,

$$
\begin{aligned}
\frac{\partial P}{\partial z} & =\frac{i \omega}{v(x, y, z)} P \\
\frac{\partial P}{\partial z} & =\frac{i \omega}{v(x, y, z)} \frac{\alpha_{1} S}{1+\beta_{1} S} P \\
\frac{\partial P}{\partial z} & =\frac{i \omega}{v(x, y, z)} \frac{\alpha_{2} S}{1+\beta_{2} S} P \\
& \cdots \cdots, \\
\frac{\partial P}{\partial z} & =\frac{i \omega}{v(x, y, z)} \frac{\alpha_{n} S}{1+\beta_{n} S} P .
\end{aligned}
$$

3-D migration or modeling involves extrapolation of the wave field using equation (1). Therefore, when using the splitting method, we need to solve the above $(n+1)$ equations in each step of extrapolation. The solution of each equation in (3) is used as boundary condition to solve for the next equation in (3), until all $(n+1)$ equations are solved for any single step of wave field extrapolation. Solving the first equation in (3) is simply a multiplication of the wave field $P$ by a phase-shift operator $\exp (i \omega / v(x, y, z))$.

The last $n$ equations in (3) all have the same form but with different constant coefficients $\alpha_{i}$ and $\beta_{i}$. Let's examine the numerical solution to a representative one of them,

$$
\frac{\partial P}{\partial z}=\frac{i \omega}{v(x, y, z)} \frac{\alpha S}{1+\beta S} P .
$$

In the $\omega-x-y$ domain, implicit finite-difference scheme: are usually used to solve the paraxial wave equation, because of their unconditional numerical stability (Clacrbout, 1985). However, direct solution of equation (4) by an implicit finite-difference schene will require solving a large $(n x \cdot n y \times n x \cdot n y)$ sparse-matrix equation, with chormons computational eff' ' (Clacrbout, 1985). A more practical but less accurate method is to use further splicting of equation (4) along inline $x$ and crossline $y$ directions 
(Brown, 1983). That is, instead of solving equation (4) in each step of extrapolation, we solve successively

$$
\begin{aligned}
\frac{\partial P}{\partial z} & =\frac{i \omega}{v(x, y, z)} \frac{\alpha S_{x}}{1+\beta S_{x}} P \\
\frac{\partial P}{\partial z} & =\frac{i \omega}{v(x, y, z)} \frac{\alpha S_{y}}{1+\beta S_{y}} P .
\end{aligned}
$$

Now, using an implicit finite-difference scheme, we solve a $(n x \times n x)$ matrix equation for different $y$ 's (difference lines) and then solve for a $(n y \times n y)$ matrix equation for different $x$ 's (difference CMP positions). The computational count in doing so is proportional to $n x \cdot n y$, a significant reduction from the direct solution method (i.c., without the $x-y$ splitting). The approximation made in the $x-y$ splitting method, however, will cause significant errors in handling steep dips, especially along diagonal lines $x= \pm y$, as analyzed in the next section.

\section{ERROR ANALYSIS AND COMPENSATION}

Equation (5) is obtained by first approximating equation (4) with the following differential equation,

$$
\begin{aligned}
\frac{\partial P}{\partial z} & \approx \frac{i \omega}{v(x, y, z)} \frac{\alpha\left(S_{x}+S_{y}\right)+2 \alpha \beta S_{x} S_{y}}{1+\beta\left(S_{x}+S_{y}\right)+\beta^{2} S_{x} S_{y}} P \\
& =\frac{i \omega}{v(x, y, z)} \frac{\alpha S_{x}}{1+\beta S_{x}} P+\frac{i \omega}{v(x, y, z)} \frac{\alpha S_{y}}{1+\beta S_{y}} P
\end{aligned}
$$

and then use splitting to separate the $x$-dependent and $y$-dependent operators. The approximation is valid only if $S_{x} S_{y}$ is zero or sufficient small.

Substituting equation (6) (with corresponding $\alpha_{i}$ and $\beta_{i}$ ) for the second, the third, ... and the last equations in (3), we recognize that equation (1) is actually replaced with the following equation,

$$
\frac{\partial P}{\partial z}=\frac{i \omega}{v(x, y, z)}\left[1+\sum_{i=1}^{n} \frac{\alpha_{i} S_{x}}{1+\beta_{i} S_{x}}+\sum_{i=1}^{n} \frac{\alpha_{i} S_{y}}{1+\beta_{i} S_{y}}\right] P .
$$

Let's define the finite-difference error $E$ as the difference between the original single square-root operator and the sum of the two split finite-difference operators plus 1 , given below,

$$
E=\sqrt{1+S_{x}+S_{y}}-\left[1+\sum_{i=1}^{n} \frac{\alpha_{i} S_{x}}{1+\beta_{i} S_{x}}+\sum_{i=1}^{n} \frac{\alpha_{i} S_{y}}{1+\beta_{i} S_{y}}\right]
$$


As will be explained later in this section, the physical meaning of $E$ is the timing error (in seconds) created per second of downward extrapolation in the finitedifference method. Given the dip angle $(\theta)$ of reflector and the inline azimuth angle $(\phi)$ (the angle between the $x$ axis and the dip direction of the reflector) as shown in Figure 1, one can extend the $S_{x}-\theta$ relation in 2-D (Claerbout, 1985) to obtain the $\left(S_{x}, S_{y}\right)-(\theta, \phi)$ relation in $3-\mathrm{D}$,

$$
\begin{aligned}
& S_{x}=(\cos \phi \cdot \sin \theta)^{2} \\
& S_{y}=(\sin \phi \cdot \sin \theta)^{2} .
\end{aligned}
$$

Therefore, $E$ can be rewritten as,

$$
\begin{aligned}
E= & \sqrt{1-\cos ^{2} \phi \sin ^{2} \theta-\sin ^{2} \phi \sin ^{2} \theta} \\
& -\left(1+\sum_{i=1}^{n} \frac{\alpha_{i} \cos ^{2} \phi \sin ^{2} \theta}{1+\beta_{i} \cos ^{2} \phi \sin ^{2} \theta}+\sum_{i=1}^{n} \frac{\alpha_{i} \sin ^{2} \phi \sin ^{2} \theta}{1+\beta_{i} \sin ^{2} \phi \sin ^{2} \theta}\right)
\end{aligned}
$$

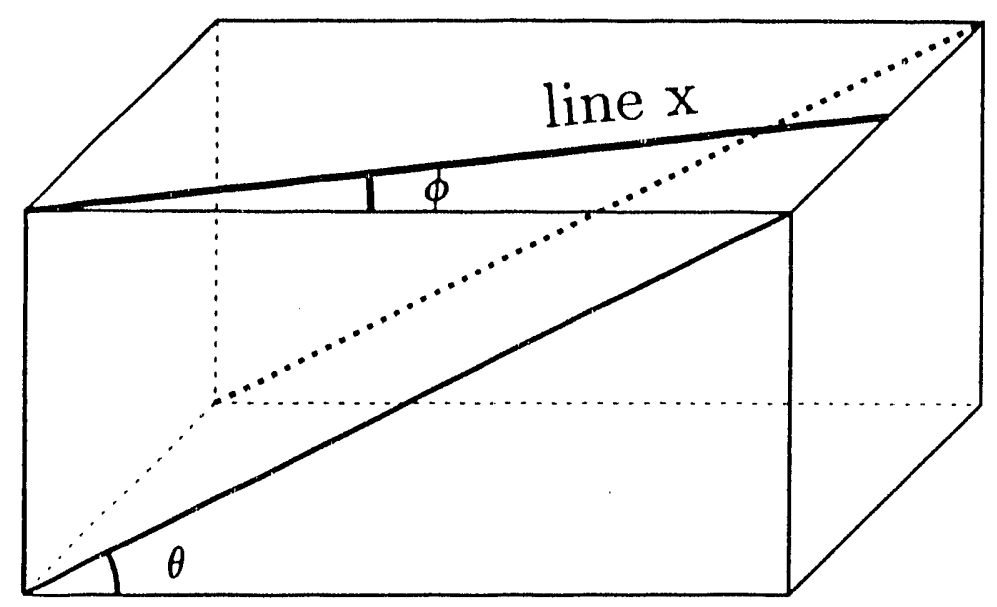

Fig. 1. 3-D model of a single dipping reflector. $\theta$ is the dip angle of the reflector. $\phi$ is the azimuth angle between line of interest and dip of reflector.

Figure 2 shows a contour plot of $E$ (when $n \rightarrow \infty$ ) as function of $\theta$ and $\phi$. When $n \rightarrow \infty, E$ accounts only for the finite-difference $x-y$ splitting error and can be written as,

$$
\begin{aligned}
E= & \sqrt{1-\cos ^{2} \phi \sin ^{2} \theta-\sin ^{2} \phi \sin ^{2} \theta} \\
& -\left(\sqrt{1-\cos ^{2} \phi \sin ^{2} \theta}+\sqrt{1-\sin ^{2} \phi \sin ^{2} \theta}-1\right) .
\end{aligned}
$$


Clearly, the inline and crossline splitting error increases as the dip angle $\theta$ increases, and is largest along the diagonal lines $x= \pm y$ ( $\phi=45$ degrees) when dip $\theta$ is fixed. The fact that the phase error varies with azimuth means that waves propagate with different velocities along different azimuth directions, a numerical anisotropy due to the inline and crossline splitting. The anisotropy of wave propagation will cause mispositioning of migrated dipping reflectors and hence misleading interpretation of complicated structures. For example, for a reflector dip of 65 degrees, the timing error after one second of downward extrapolation of the surface data will be $123 \mathrm{~ms}$.

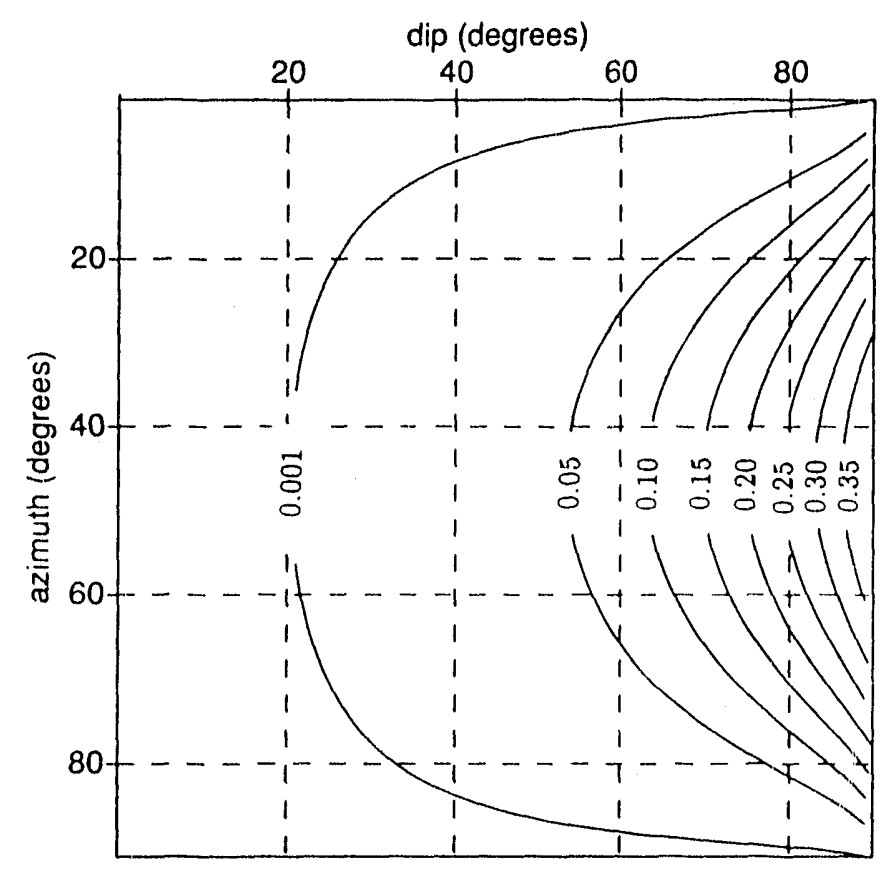

FIG. 2. Contour plot of inline and crossline splitting error. The splitting crror is defined as the time error (s) per one second of downward extrapolation.

To compensate for the finite-difference errors and yet still retain the efficiency of the splitting method, we need to solve an extra phase-compensation equation at each step of wave field extrapolation,

$$
\frac{\partial P}{\partial z}=\left[\frac{i \omega}{v(x, y, z)} E\right] P
$$

The finite-difference error compensation equation in (12) can be solved using any of several familiar numerical methods used to solve wave equations. The square-root operator in $E$ have to be expanded and approximated to a certain order of paraxial equation, if the finite-cifference method is to be used. For example, expanding the operator $E$ in equation (8) and ignoring higher-order terms gives the first-order paraxial equation for the error compensation, 


$$
\frac{\partial P}{\partial z}=-i \frac{v^{3}(x, y, z)}{\omega^{3}} \frac{\partial^{2}}{\partial x^{2}} \frac{\partial^{2}}{\partial y^{2}} P .
$$

Solving equation (13) using a first-order forward explicit finite-difference scheme along the $z$ axis will result in an unconditionally unstable solution, because the norm of the transfer function is alway greater than or equal to 1. Damping of growing amplitudes must be applied in order to ensure stability (Graves and Clayton, 1990), if the explicit scheme is to be used. Implicit schemes (without $x-y$ splitting), on the other hand, though unconditionally stable, require relatively heavy computation, which we tried to avoid by using the $x-y$ splitting method to solve equation (4) in the first place.

Since the error $E$ is small in a single step of wave field extrapolation, the effect of the compensation process is similar to that of residual migration (Rothman, 1985), in that waves propagate very little in one extrapolation step. Therefore, when lateral velocity variation is moderate, it is reasonable to use a reference velocity $v_{a}(z)$ (for example, velocity averaged over $(x, y))$ to replace $v(x, y, z)$ in equation (12) and thus benefit from a phase-shift solution,

$$
P(z+\Delta z)=P(z) \exp \left(\frac{i \omega}{v_{a}(z)} E \Delta z\right) .
$$

Since a phase-shift operator which is a linear function of frequency $\omega$ corresponds to a time shift in the time domain, we recognize that $E$ is actually the timing error of the finite-difference splitting for one $\Delta z / v$ (or timing error per one second of downward extrapolation).

It turns out that my approach is similar to that of Kitchenside (1988). Kitchenside implemented the first square root operator (the 3-D wave equation) in equation (8) with the phase-shift migration using a minimum velocity $v_{\alpha}(z)$. He then combined the remaining operators in equation (8) (using velocity $v_{\alpha}(z)$ ) with the operators in equation (1) (using velocity $v(x, y, z)$ ) to obtain a residual wave-field extrapolation equation. Instead of using the implicit finite-difference method, he solved the residual wave-field extrapolation equation by the explicit finite-difference method. Since a laterally invariant velocity function is used in implementing the operators in equation (8), naturally, I choose to use the accurate phase-shift operator for every operator in equation (8), which leaves the migration part of solving equation (3) unchanged from the conventional implicit finite-difference method. One major advantage of my approach is that the commonly-used, conventional, 3-D finite-difference migration need not be changed and the error compensation only applies as a phase-shift filter at certain depth steps. Both Kitchenside's and my approaches will have the accuracy of the phase-shift migration (i.e., without steep-dip limitation, no $x-y$ splitting error, and no finite-difference dispersion), when velocity is a function of depth only. When velocity varies laterally, we both can use Gazdag's method of phase-shift plus interpolation (Gazdag and Sguazzero, 1984) to get better accuracy. However, since I use the phase-shift method to solve the residual phase-error compensation equation (12) while Kitchenside uses the phase-shift method to solve the wave equation (1), the 
error of my approach, using the phase-shift plus interpolation, shall be smaller than that of Kitchenside's approach, when velocity varies laterally. As will be explained later, the residual error compensation can be applied every few depth steps of extrapolation while the 3-D wave equation in Kitchenside's approach has to be solved every depth step, therefore, my approach is also more efficient than that of Kitchensicle.

It is important that all the aspects, including the Crank-Nicolson scheme (Clacrbout, 1985), the finite-difference approximation of derivatives (Clacrbout, 1985), and the so-called $1 / 6$ trick (Clacrbout, 1985), of the conventional implicit finite-difference solution to equation $(3)$ be taken into consideration when solving equation (12). After some algebra, the solution to equation (12), if the conventional implicit finitedifference method is used in migration, is given by,

$$
P(z+\Delta z)=\exp \left[i\left(k_{z}-\frac{\omega}{v_{a}(z)}\right) \Delta z\right] \times \prod_{j=1}^{n} \frac{a_{j}+i i_{j}}{a_{j}-i b_{j}} \times \prod_{j=1}^{n} \frac{c_{j}+i d_{j}}{c_{j}-i d_{j}} \times P(z)
$$

where,

$$
\begin{aligned}
& k_{z}=\sqrt{\left(\frac{\omega}{v_{a}(z)}\right)^{2}-k_{x}^{2}-k_{y}^{2}}, \\
& a_{j}=\frac{1-\left[\gamma \Delta x^{2}+\beta_{j}\left(\frac{v_{a}(z)}{\omega}\right)^{2}\right] \hat{k}_{x}^{2}}{\Delta z}, \\
& b_{j}=\frac{v_{a}(z) \alpha_{j} \hat{k}_{x}^{2}}{2 \omega}, \\
& c_{j}=\frac{1-\left[\gamma \Delta y^{2}+\beta_{j}\left(\frac{v_{a}(z)}{\omega}\right)^{2}\right] \hat{k}_{y}^{2}}{\Delta z}, \\
& d_{j}=\frac{v_{a}(z) \alpha_{j} \hat{k}_{y}^{2}}{2 \omega} .
\end{aligned}
$$

$\gamma$ in equation (16) is the so-called 1/6-trick value used to improve the accuracy of using the second-order finite-difference to approximate the second spatial derivative, with typical $\gamma$ value of $0.14 . \hat{k}_{x}^{2}$ and $\hat{k}_{y}^{2}$ are approximations to the true lateral wavemumbers that result from the second-order finite-difference approximations of the derivative operators $-\partial^{2} / \partial x^{2}$ and $-\partial^{2} / \partial y^{2}$, respectively, as expressed below (Claerbout, 1985),

$$
\begin{aligned}
& \hat{k}_{x}^{2}=\frac{2-2 \cos \left(k_{x} \Delta x\right)}{\Delta x^{2}}, \\
& \hat{k}_{y}^{2}=\frac{2-2 \cos \left(k_{y} \Delta y\right)}{\Delta y^{2}} .
\end{aligned}
$$

Figure 3 shows the impulse response of the finite-difference-error compensation operator computed by the phase-shift method, for a frequency of $20 \mathrm{~Hz}$ and a depth 
step of $100 \mathrm{~m}$. The operator is anisotropic, with maximum data adjustment along the diagonal lines and no action along either $x=0$ or $y=0$ lines. The effective area over which the operator applies become smaller as frequency becomes higher and as the depth step becomes smaller. In practice, the error compensation operator need be applied only once every few depth extrapolation steps. Because of the narrowness of the effective width of the operator, a 2-D convolutional method, can also be used efficiently to handle lateral velocity variation, but caution must be taken to avoid numerical instability.

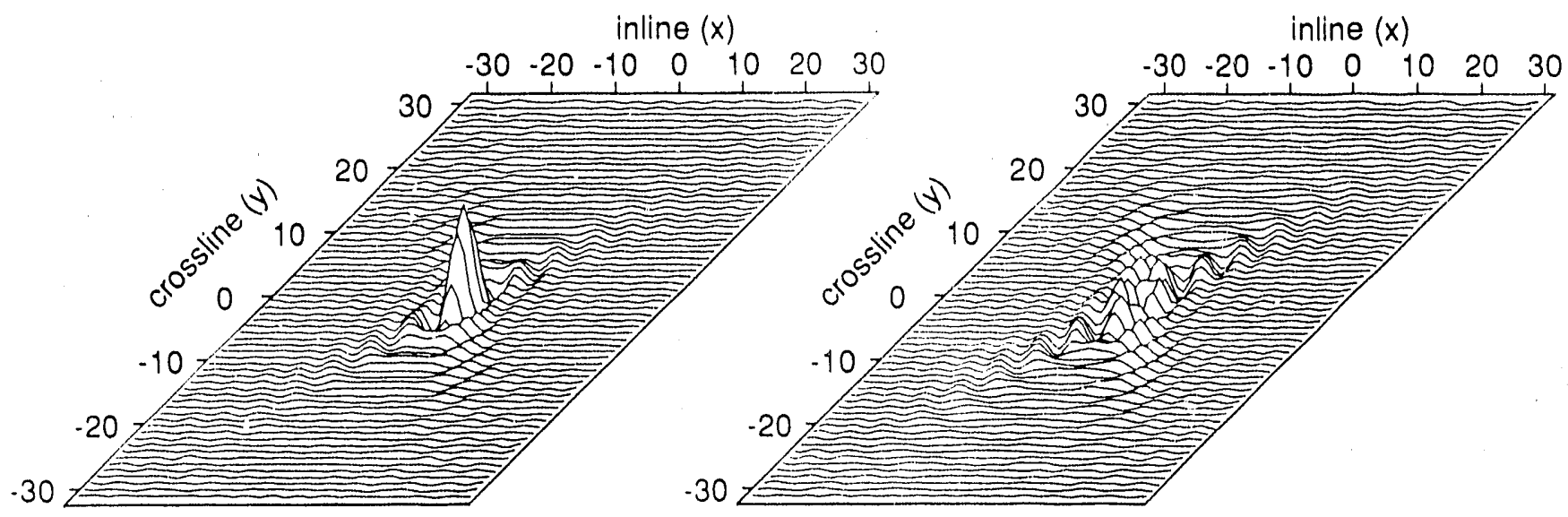

(a)

(b)

FIG. 3. Impulse response of the finite-difference splitting-error compensation operator for a frequency of $20 \mathrm{~Hz}$ and a compensation step of $100 \mathrm{~m}$. Sampling intervals along $x$, $y$ and $z$ are $12.5 \mathrm{~m}$. Velocity is constant, $2000 \mathrm{~m} / \mathrm{s}$, in the model. Data manipulation takes place mostly along the diagonal directions of the $x-y$ plane, where $x$ is the inline coordinate and $y$ is the crossline coordinate. (a) real part of the response; (b) imaginary part of the response.

The accuracy of using equation (14) with one reference velocity $v_{a}$ to compensate for the finite-difference splitting errors in the presence of lateral velocity variation is the same as that of using Kitchenside's method. When lateral velocity variation is large, Gazdag's method of phase-shift plus interpolation (Gazdag and Sguazzcro, 1984) can be used to solve equation (12). Defining $n_{a}$ to be number of reference velocities used to solve equatic (14), and $A_{i}$ and $\Theta_{i}$ to be the amplitude and the phase of the solution $P_{i}(z+\Delta z)$ to equation (14) using reference velocity $v_{i}$, we can then use polynomial interpolation of the $n_{a}$ individual solutions $P_{i}(z+\Delta z)$ to obtain the solution $P(z+\Delta z)=A \exp (i \Theta)$ at location $(x, y, z)$, 


$$
\begin{aligned}
& A=\operatorname{polint}\left(V_{a}^{3}, A_{a}, n a, v^{3}(x, y, z)\right), \\
& \Theta=\operatorname{polint}\left(V_{a}^{3}, \Theta_{a}, n a, v^{3}(x, y, z)\right),
\end{aligned}
$$

where polint is the polynomial interpolation function, $V_{a}=\left(v_{1}(z), v_{2}(z), \ldots, v_{n_{a}}(z)\right.$ ) is the reference velocity vector, $A_{a}=\left(A_{1}, A_{2}, \ldots, A_{n_{a}}\right)$ is the amplitude vector and $\Theta_{a}=\left(\Theta_{1}, \Theta_{2}, \ldots, \Theta_{n_{a}}\right)$ is the phase vector. The interpolation is performed along $v_{a}^{3}$ axis because, as indicated in equation (13), the leading term in the phase error due to the finite-difference splitting is proportional to $v^{3}$.

Figure 4 shows the percentage of root-mean-squared (rms) relative phase error (after applying the constant-velocity phase-shift compensation) as a function of percentage of lateral velocity variation, along the diagonal line ( $\phi=45$ degrees) with dip angle of 45 degrees, for $n_{a}=1,3$ and 5. The rms relative phase error $p_{r m s}$ is defined as

$$
p_{r m s}=\sqrt{\frac{1}{\left(v_{\max }-v_{\min }\right)} \int_{v_{\min }}^{v_{\max }}\left[\frac{p(v)-p_{s p l i t}(v)-p_{c o m p}(v)}{p(v)}\right]^{2}} d v
$$

where $v_{\max }$ is the maximum velocity, $v_{\min }$ is the minimum velocity, $p(v)$ is the correct phase computed using the wave equation $(1), p_{\text {split }}(v)$ is the phase computed using the splitting equation $(7), p_{\text {comp }}(v)$ is the phase interpolated from the $n_{a}$ phases computed from the compensation equation (14). The lateral velocity variation $v_{\text {lat }}$ is defined as,

$$
v_{\text {lat }}=\frac{v_{\max }-v_{\min }}{v_{\min }} .
$$

The original percentage error using the conventional splitting method, in this case, is 3.53. Therefore, as shown in Figure 4, even for 100 percent lateral velocity variation, the relative phase error is 2.5 percent if only one constant-velocity phase-shift compensation (i.e., without interpolation) is used, still a reduction of 30 percent of phase error from the conventional finite-difference splitting method (without the phase compensation). The relative phase error drops to 0.35 percent, if five reference velocities are used in the phase compensation to give the interpolated phase. Figure 4 helps us determine the number of reference velocities needed, for given acceptable phase error and given laieral velocity variation.

In media of strong lateral velocity variation, the 3-D migration with my approach of compensating finite-difference splitting error has higher accuracy than Gazdag's method of phase shift plus interpolation, because interpolation is applied to the computation of the residual phase error ( that is much smaller than the phase itself) while Gazdag applied interpolation to the compuration of the phase. If the residual phase error compensation is done every 10 depth steps with three reference velocities, the cost of 3-D migration using my method will be that of the conventional 3-D finitedifference migration plus $3 / 10$ of that of single-velocity $3-D$ phase-shift migration. 


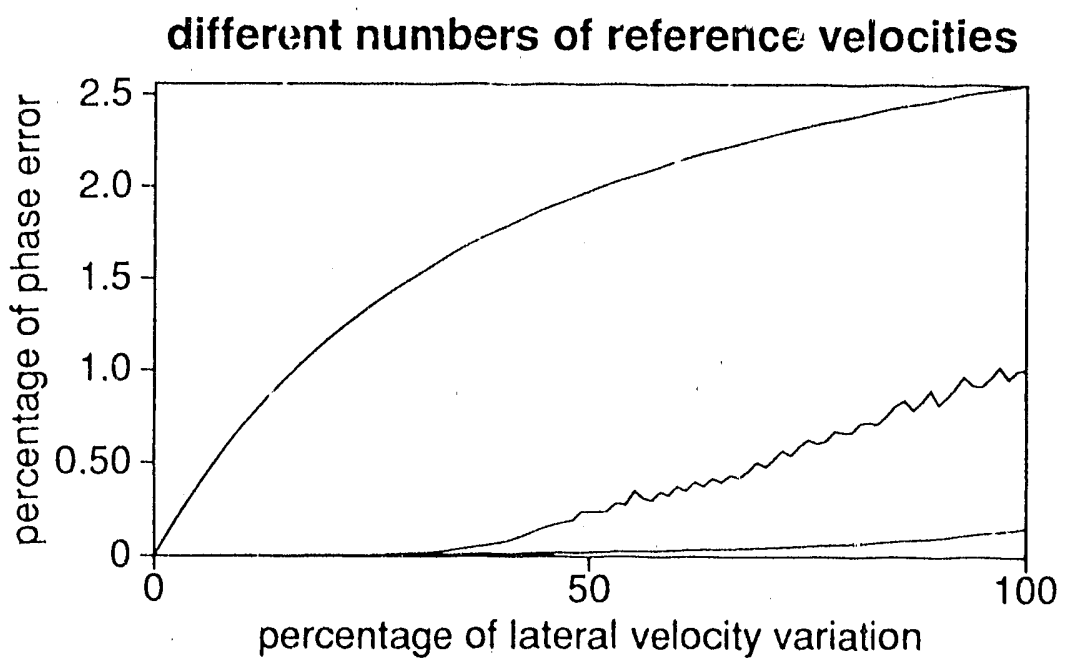

FIg. 4. Percentage of the remaining phase error as a function of percentage of lateral velocity variation for azimuth angle of 45 degrees and dip angle of 45 degrees. Number of reference velocities used in the crror compensation, shown in the highest, the middle, and the lowest curves, is one, three and five, respectively.

\section{IMPLEMENTATION AND EXAMPLES}

3-D poststack migration downward continues tne input CMP stack and obtains the migrated images from the downward extrapolated wave field at $t=0$. During each step of downward extrapolation of the wave field, the first equation in (3) is solved first, next, the last $n$ equations in (3) are solved sequentially using the $x-y$ splitting method, and, then, every few depth steps the finite-difference-error compensation equation (12) is solved using the phase-shift method. In the frequency-space domain, one-pass 3-D depth migration with finite-difference-error compensation is implemented as follows.

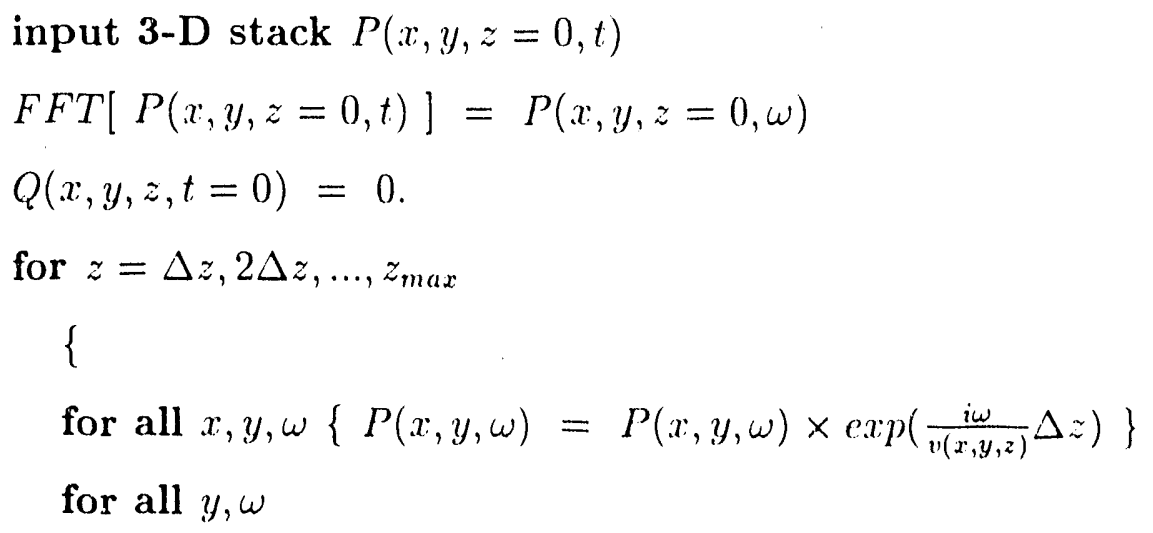




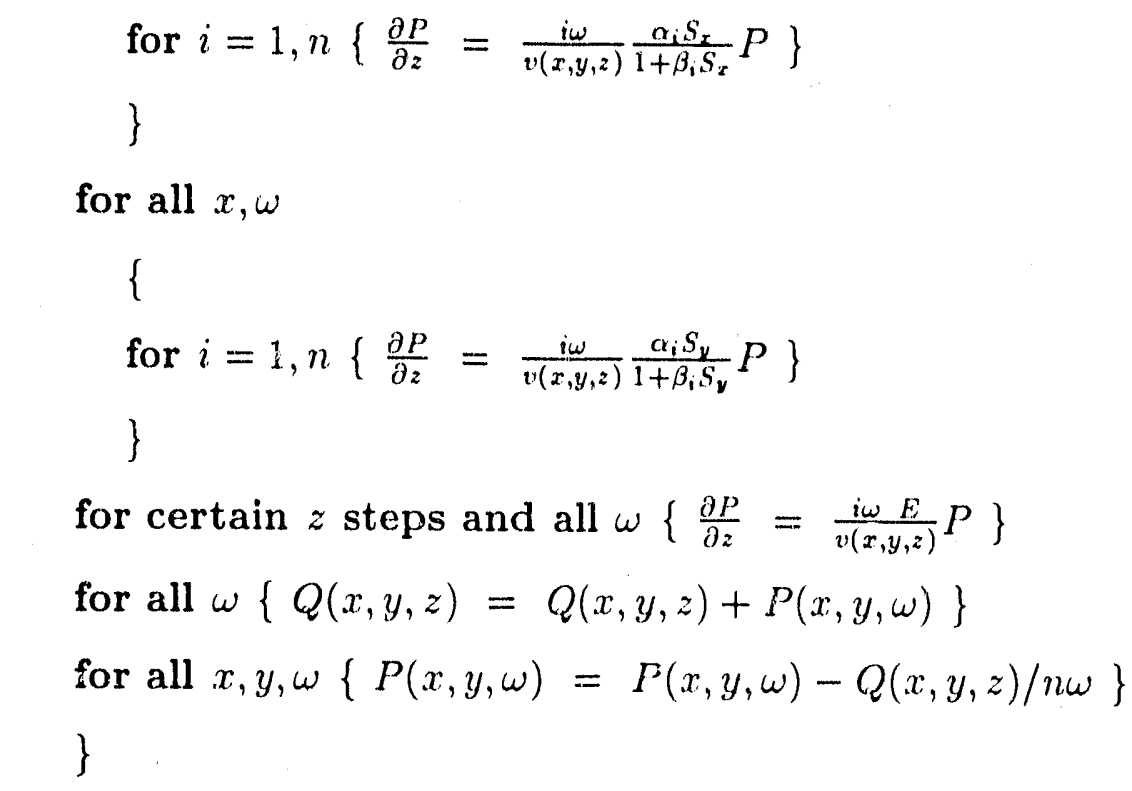

output 3-D migrated data $Q(x, y, z)$

As seeu here, the image is obtained by summing the downward-continued wave field along the $\omega$ axis, giving the wave field at $t=0$. The subtraction of in.age $Q$ from the downward extrapolated wave field $P$ in the last step of each downward extrapolation step reduces the FFT wrapround along the time axis (Kjartansson, 1979).

Similarly, implementation of 3-D poststack forward modeling in the frequencyspace domain is as follows.

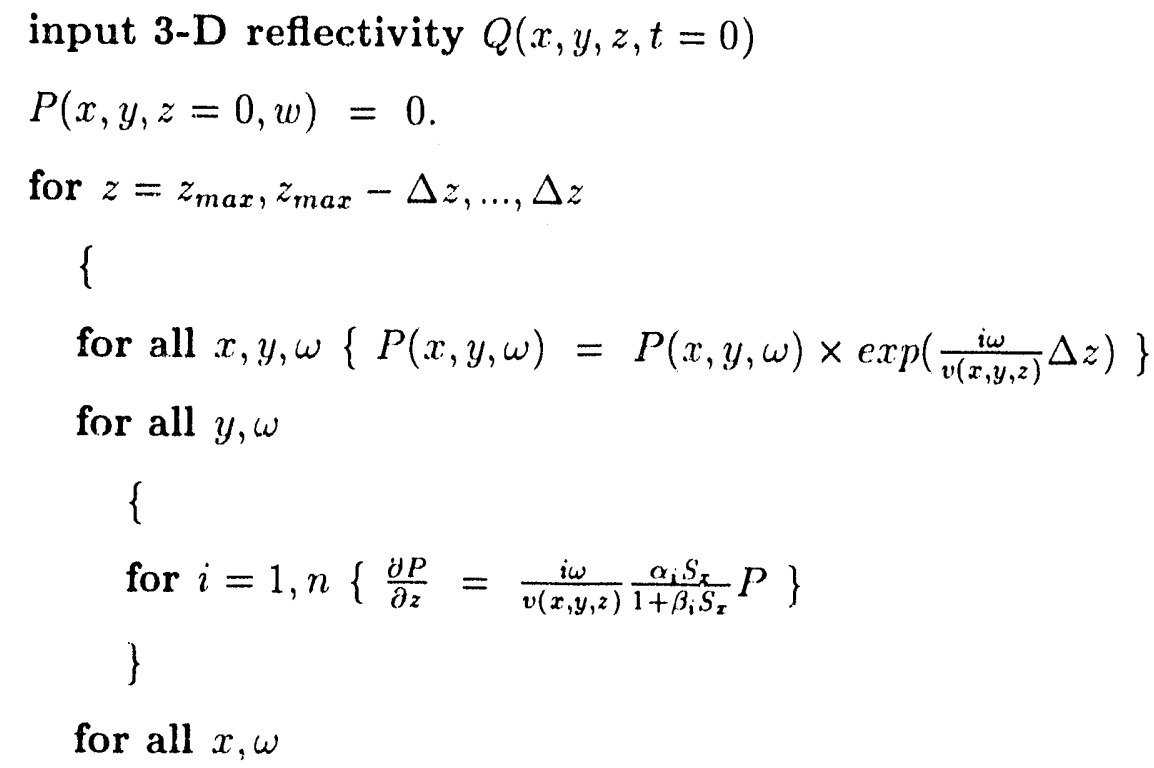




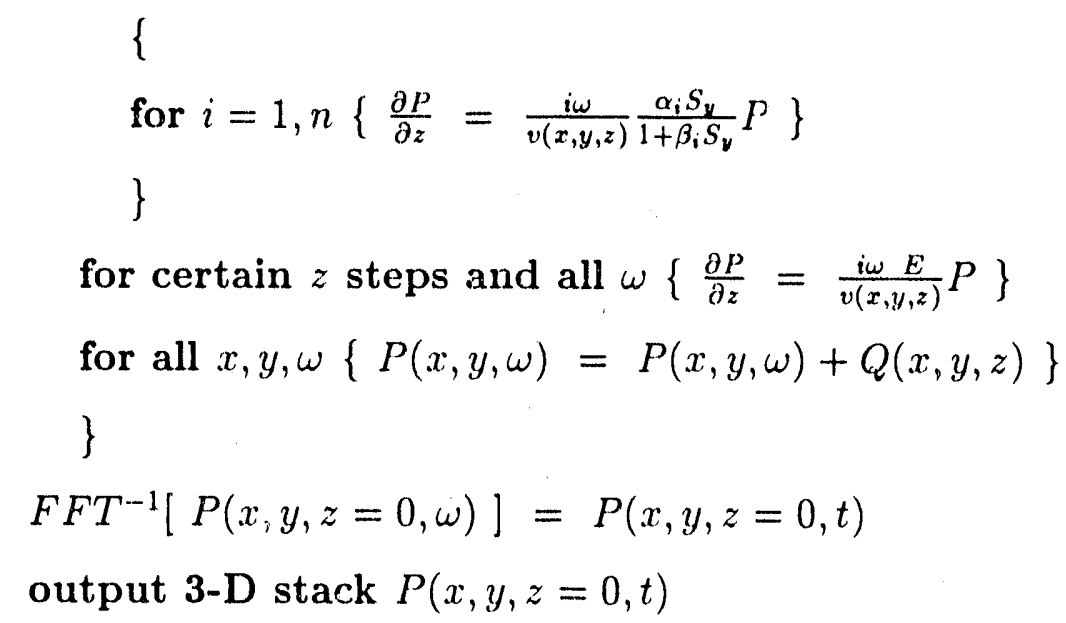

Instead of summing the downward extrapolated wave field along the frequency axis as when doing the 3-D migration, the reflectivity function $Q$ is added to the upward continued wave field $F$ at each depth level to become exploding sources at $t=0$. The surface-recorded, 3-D poststark data are obtained from the wave field upward extrapolated to $z=0$.

Because the finite-difference splitting error in each depth step is small, though cumulative error may be large, the error compensation can be applied every few depth steps of extrapolation to reduce the computational effort of the compensation process. With the compensation step being eight depth-extrapolation steps, tests showed that the error compensation process increases the total computational cost by about 15 percent.

Figure 5 compares impulse responses of 3-D migration without and with the error compensation. An impulse is placed at $x=y=0$ and at time $t=28$ in the input 3-D stack. A migration operator with order $2 n=2$ is used in both tests. As expected, the conventional 3-D migration (without error compensation) gives a result that departs from the ideal - a hemisphere. The depth slices of the conventional approach display diamond-shape (as opposed to the correct circular) responses caused by the anisotropy of the finite-difference splitting method. Note also the build up of evanescent energy near the center of the impulse response. This evanescent energy becomes dominant at shallow depth slices. With phase-shift implementation of the finite-difference error compensation operator, on the other hand, the 3-D migration gives a more nearly circular and correctly positioned impulse response. Also, as shown in Figure 5. because the phase-shift method propagates only the nonevanescent energy, the error compensation has the additional advantage of suppressing evanescent energy generated by the finite-difference implementation of wave equation migration. Furthermore, the accuracy of imaging steep dips is improved to 90 degrees, since the velocity in the model is constant.

Figure 6 compares impulse responses of 3-D modeling without and with finitedifference error compensation. An impulse is specified at $x=y=0$ and at $z=12 \mathrm{in}$ 


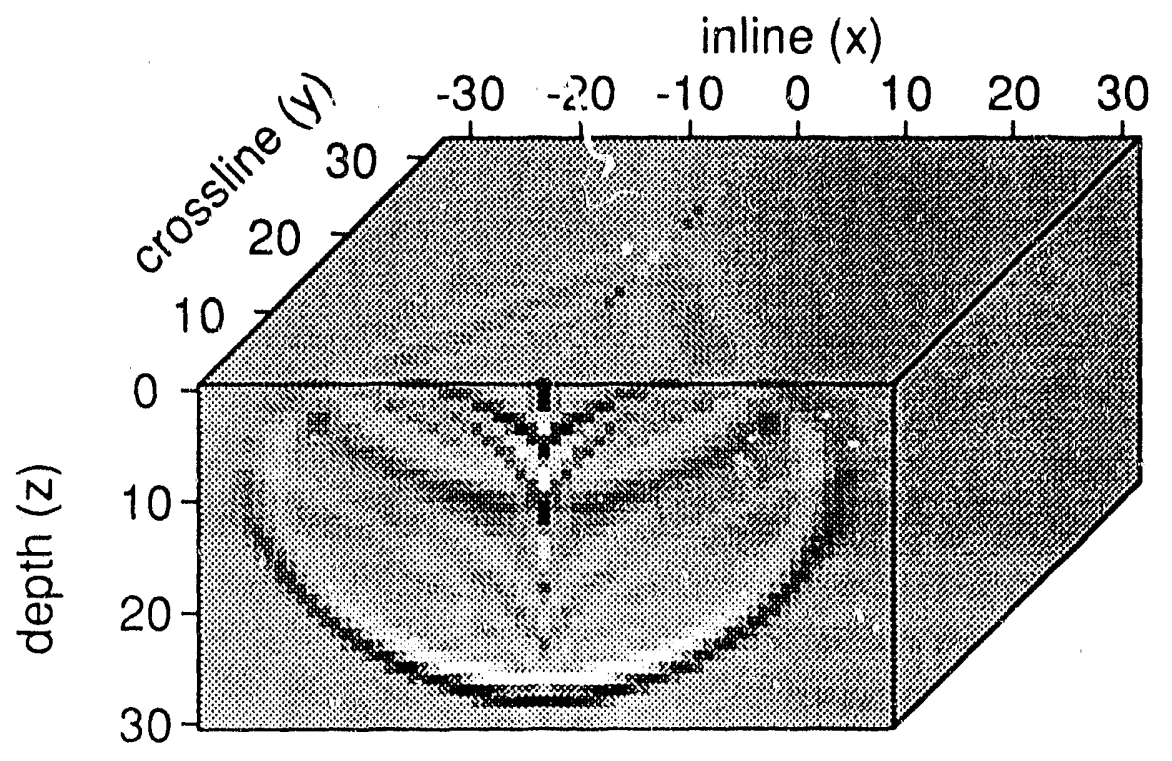

(a)

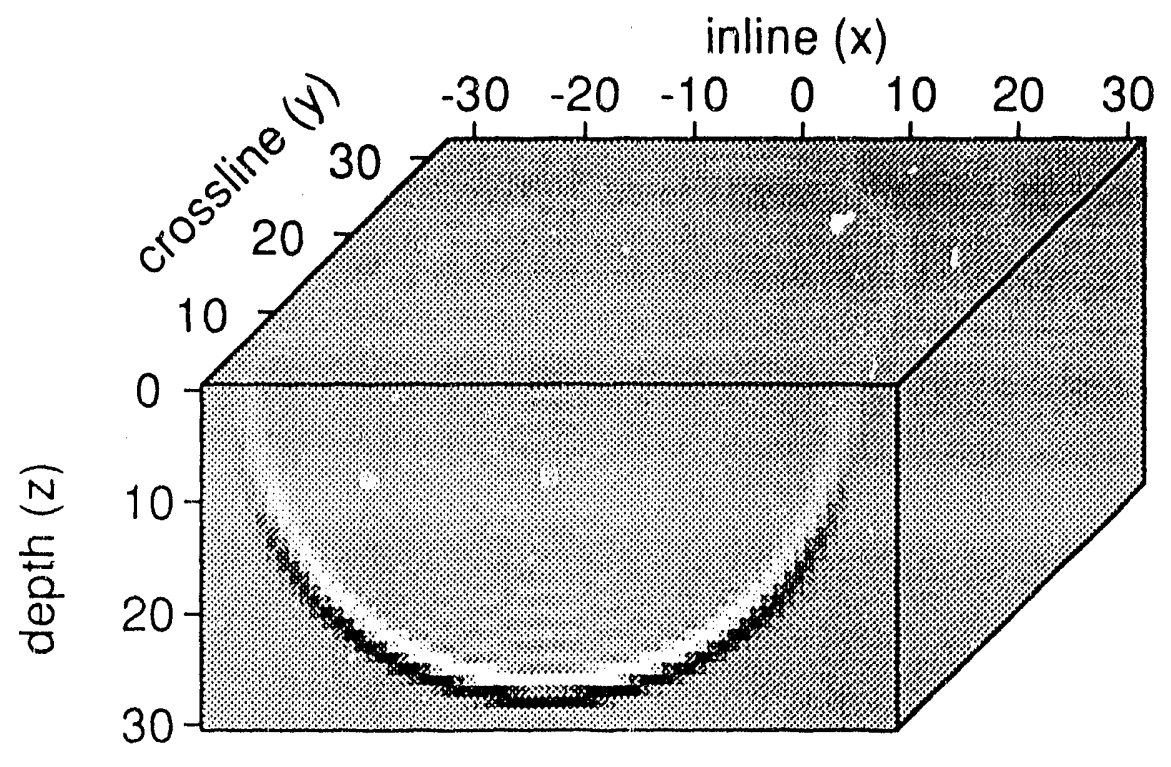

(b)

FIG. 5. Comparison of impulse responses of 3-D migrations without [(a), (c), and (e)] and with $[(b),(d)$, and (f)] the finite-difference error compensation. Sampling intervals along $x, y, z$ and $t$ are 1 , with constant velocity $v=2$, in the computation. A 65-degree extrapolation operator is used in both cases. (a) and (b): cube display of half volume of 3-D impulse response. 


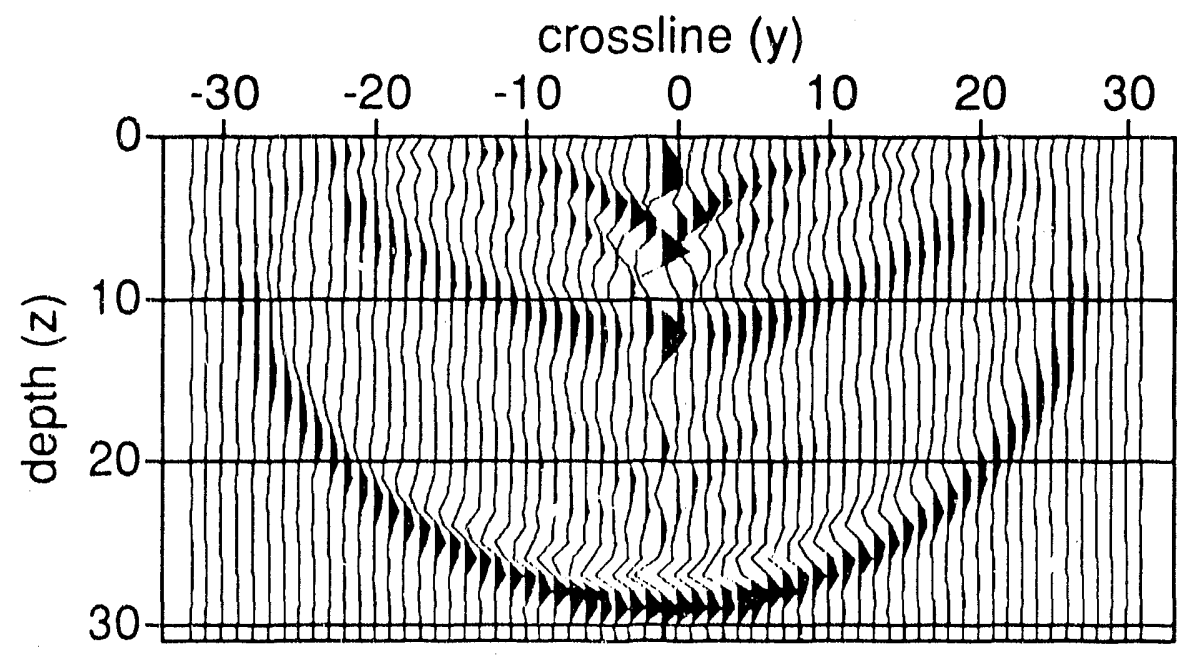

(c)

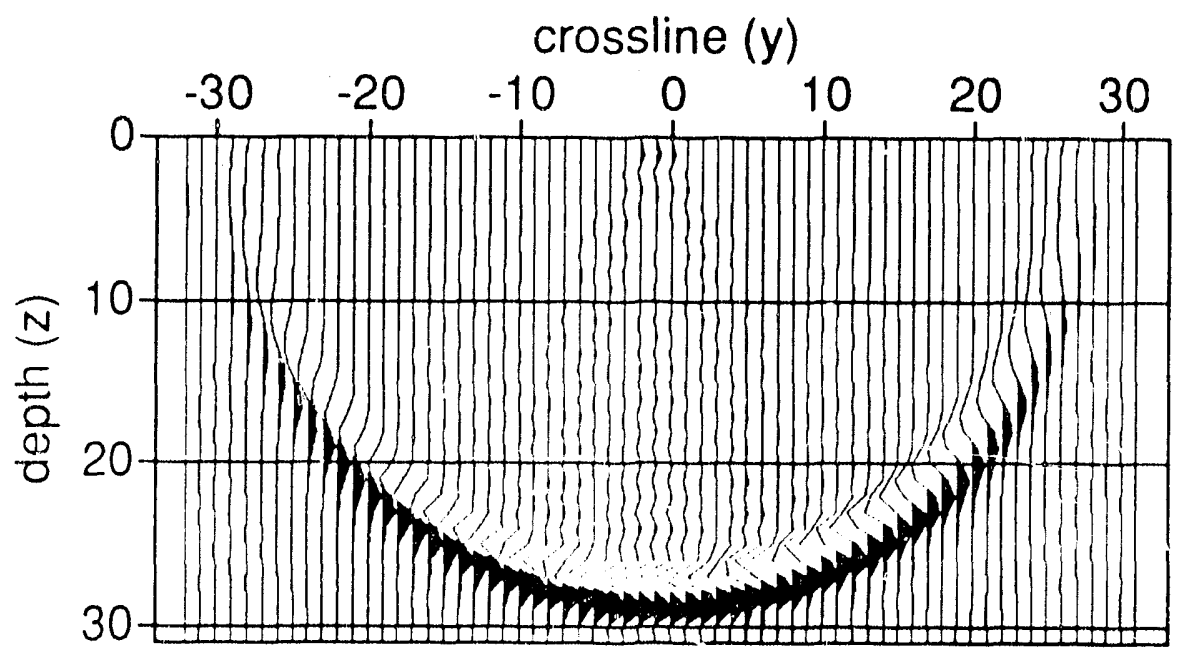

(d)

FIG. 5. (c) and (d): vertical section at $x=0$. 

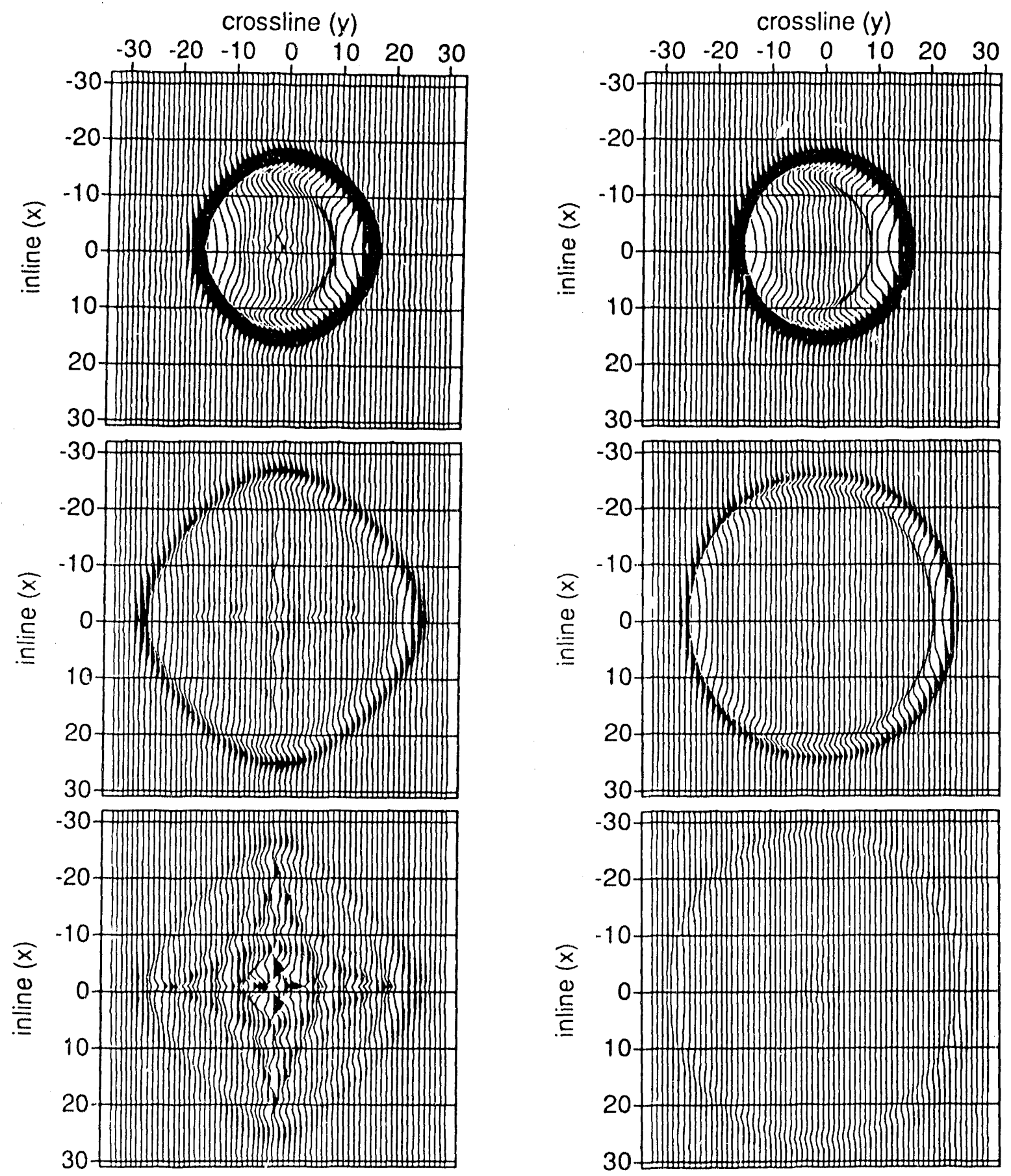

(e)

(f)

FIG. 5. (c) and (f): depth slices at $z=25, z=15$ and $z=5$. 
the input 3-D reflectivity model. Again the paraxial equation of order $2 n=2$ is used in both cases. Figures $6(s)$ and $6(d)$ show the diffractions generated with the two approaches along the diagonal line $x=y$. The error in arrival time of the diffraction at the edges is as large as about 10 time samples, though the total travel time is about 45 time samples, a relative crror of more than 20 percent! With the finite-differencecrror compensation, the impulse response of the 3-D modeling is more accurate and has less evanescent energy present than does that of the conventional approach.

A more geologically plausible model is tested and results are shown in Figure 7. The model has four reflectors, with the medium velocity varying linearly with depth, $n(z)=15(0)+2 \times z(\mathrm{~m} / \mathrm{s})$. The first reflector is an upward hemisphere truncated with a lorizontal bed. The strikes of the two dipping interfaces, with dips of 45 degrees and 60) degrees, respectively, are perpendicular to the diagonal line $x=y$. Both dipping interfaces are truncated with horizontal beds. The fourth reffector is simply horizontal. The 3-D phase-shift method is used in forward modeling of the wave ficld. 3-D frequency-space depth migration of order $2 n=2$ withont and with finitedifference-erre: compensation is used in migration of the 3-D stack. Figure 7 displays six rows of pictures, with four pictures in each row. The pictures in each row are, in order, the reflector morlel, 3-D phase-shift modeling, conventional 3-D frequencyspace depth migration without cror compensation, and 3-D frequency-space depth migration with error compensation. The migration with the compensation gives more accurate images and higher dip accuracy of the hemisphere than does the anigration without the compensation, as shown in Figures $7(b), 7(\mathrm{c})$ and $7(\mathrm{c})$. In Figure $7(\mathrm{~d})$, vertical sections at $x=0$ show that the 60-degrec dipping reflector is undermigrated and weakened in the migration without the compensation. The anisotropy of the 3-D migration due to the inline and crossline splitting gives the diamond-shape image of the original circle on the depth slice of migrated 3-D data, as shown in Figure 7(c). The anisotropic error is suppressed by the error compensation process.

\section{CONCLUSION}

The accuracy of conventional one-step, $x-y$ splitting, $3-\mathrm{D}$ depth migration and modeling can be improved by doing the finite-difference-error compensation during the wave field extrapolation. When lateral velocity variation is moderate, the compensation can be simply done using phase-shift method. The modified 3-D wave field extrapolation method retains the efficiency of the splitting approach, yet overcomes problems of mispositioning of steep dip events and creating undesirable dispersion and exanescent energy in the conventional 3-D wave field extrapolation method.

Phase-shift plus interpolation method, or other numerical methods, such as the 2-D convolutional method with a stable convolution operaton, must be used to solve the compensation equation, when strong lateral velocity variations are present in the arca. 


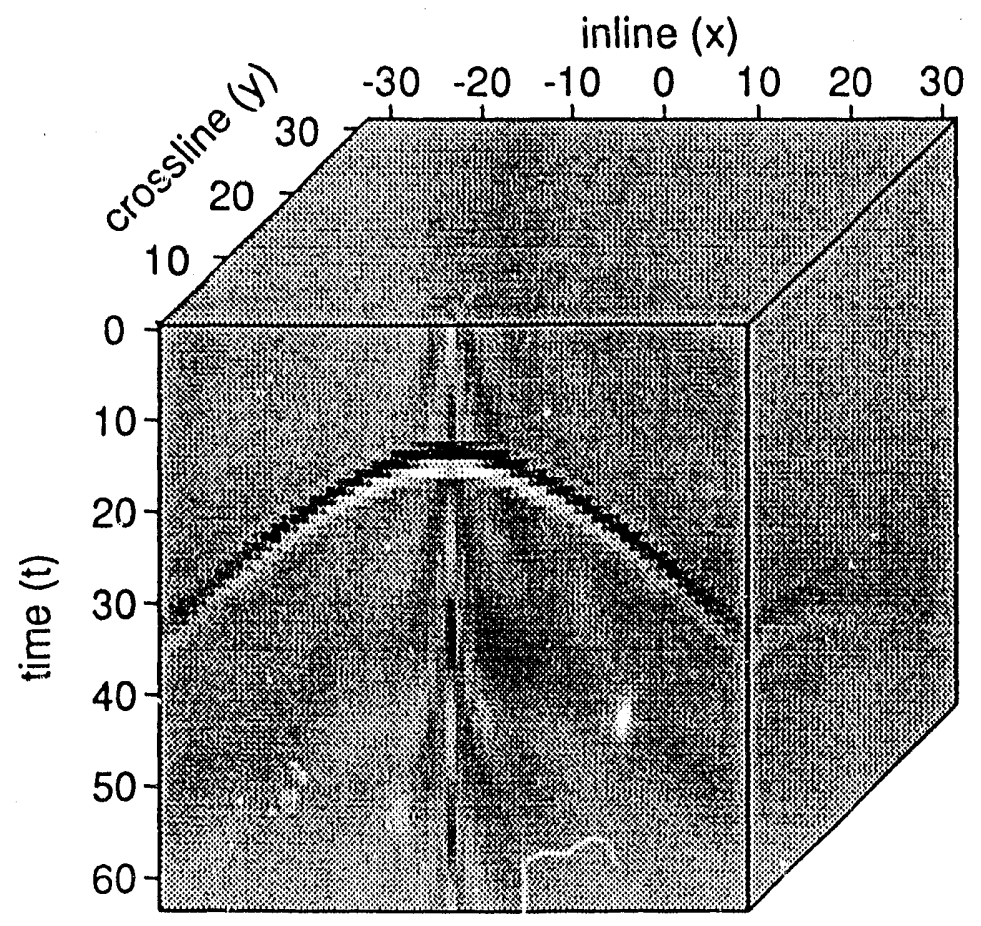

(a)

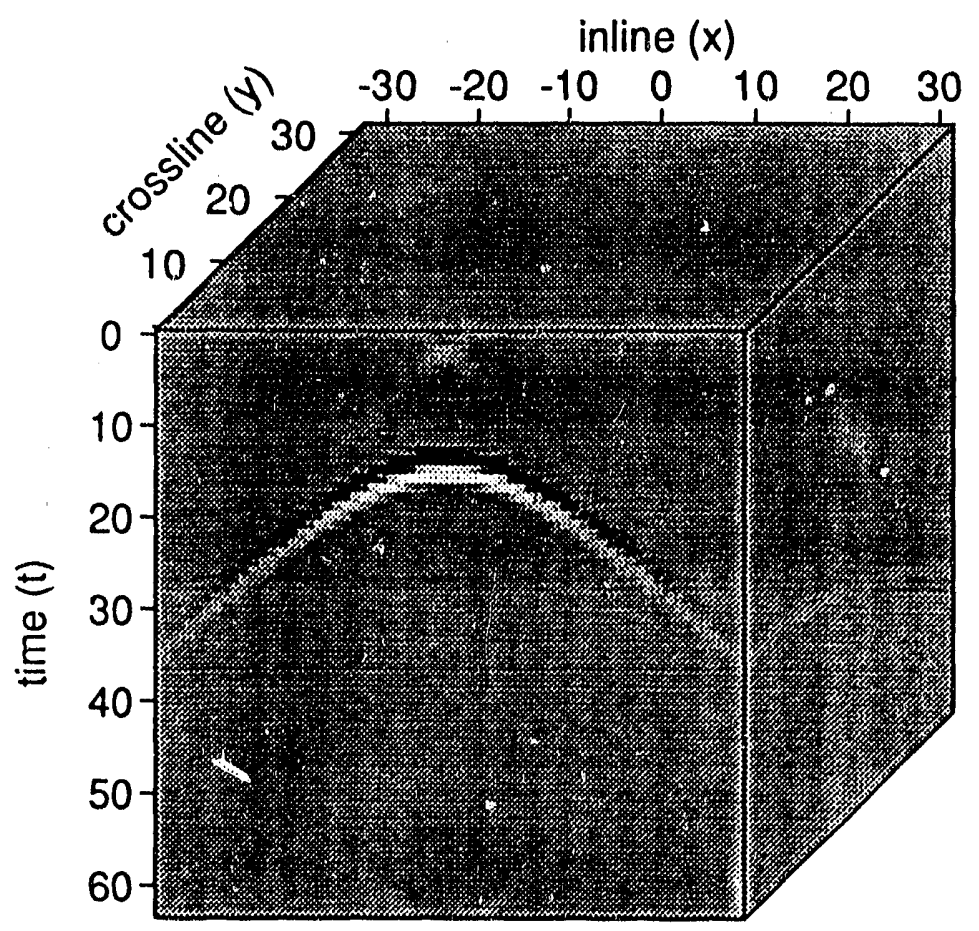

(b)

FIG. 6. Comparison of impulse responses of 3-D modeling without [(a), (c), and (e)] and with [(b), (d), and (f)] the finite-difference error compensation. Sampling intervals along $x, y, z$ and $t$ are 1 , with constant velocity $v=2$, in the computation. A 65-degree extrapolation operator is used in both cases. (a) and (b): cube display of half volume of 3-D impulse responses. 


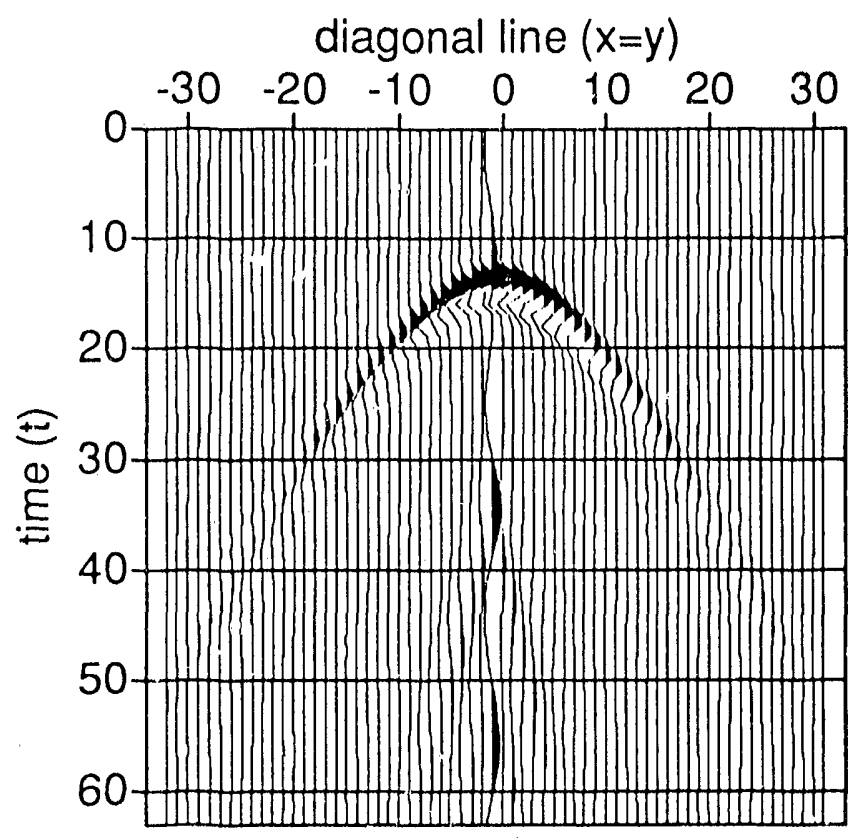

(c)

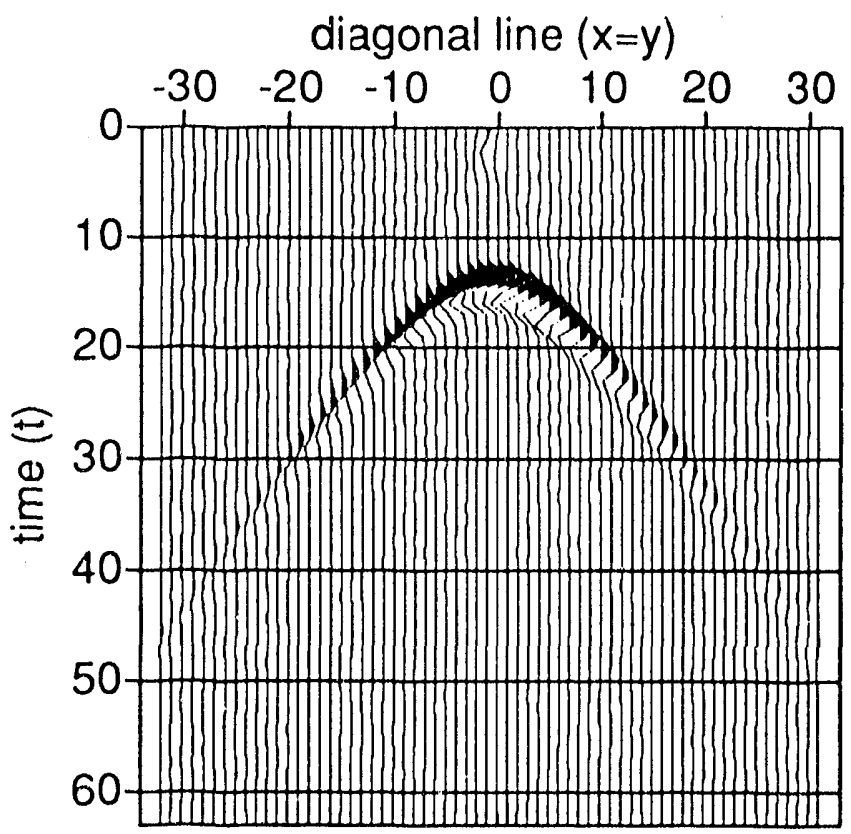

(d)

Fig. 6. (c) and (d): vertical section at diagonal line $x=y$. The error is maximum along $x= \pm y$ lines. The timing errors of the diffraction at both edges in (c) are as large as 10 simules. 


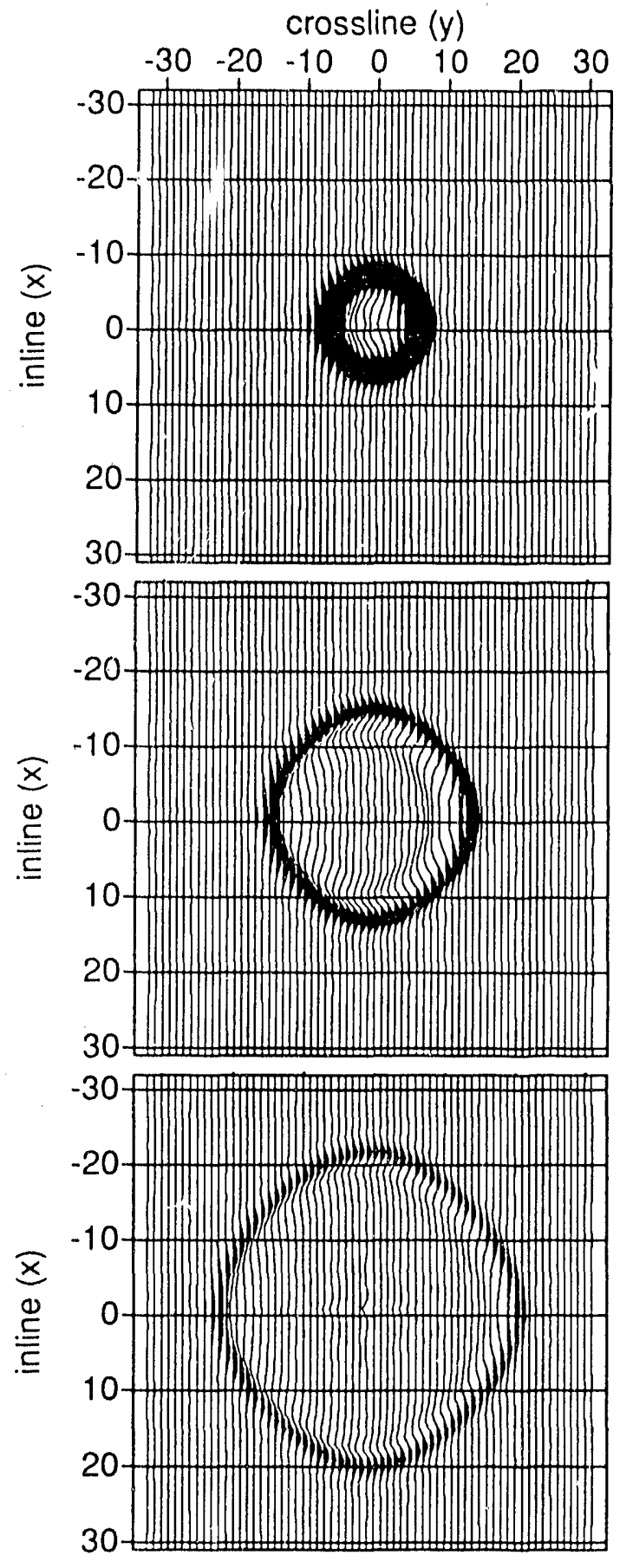

(e)

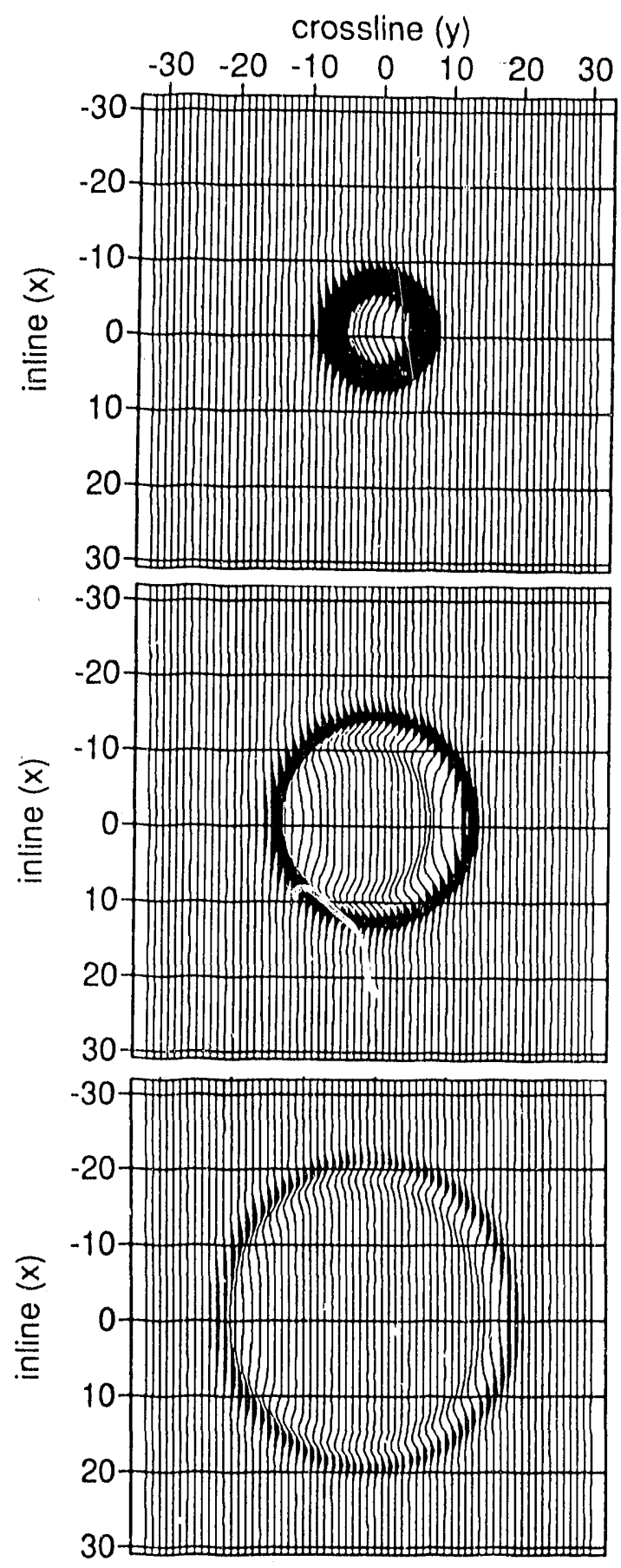

(f)

FIG. 6. (e) and (f): time slices at $t=15, t=20$ and $t=25$. 

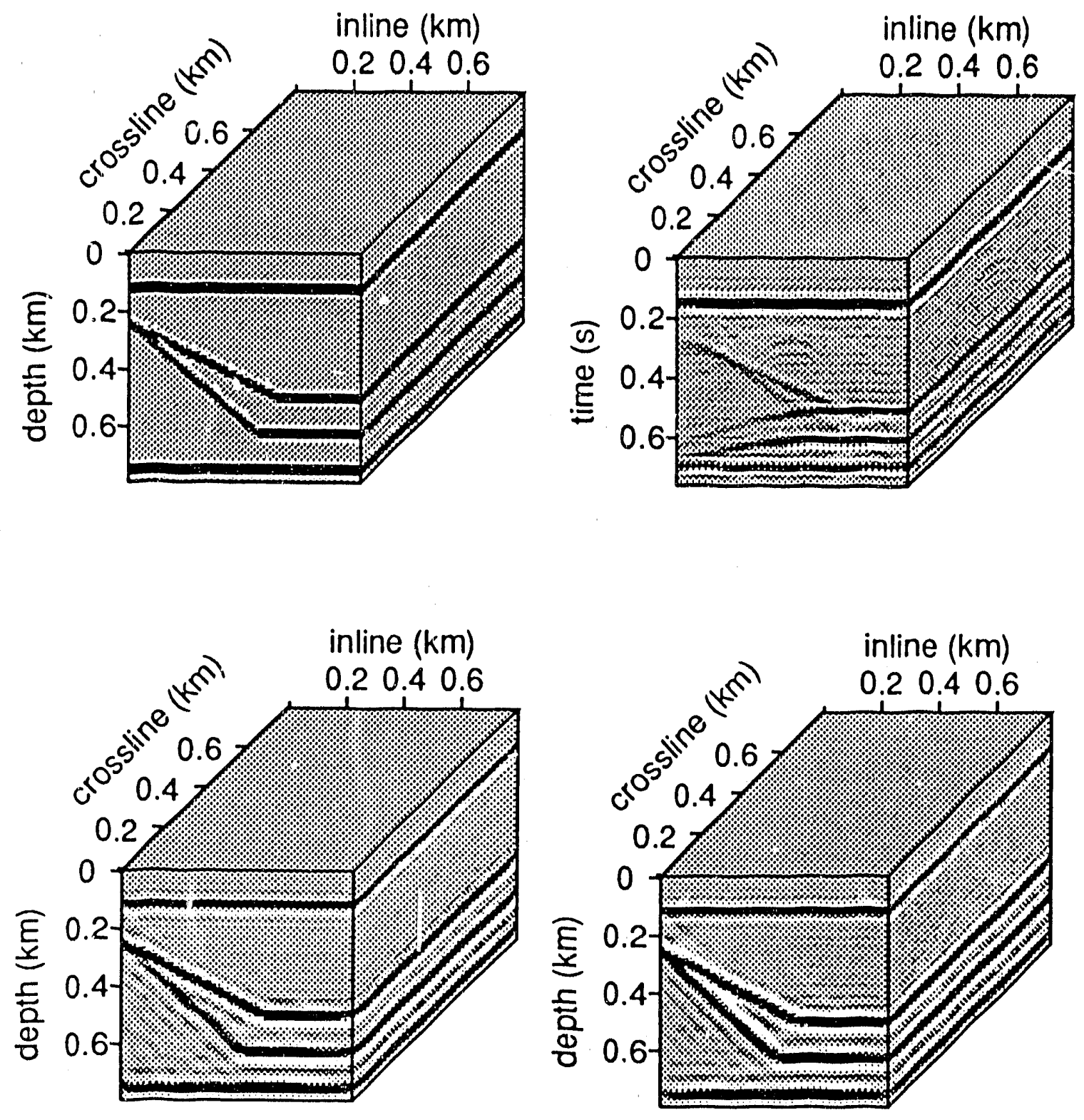

(a)

FIG. 7. Comparison of 3-D migration without and with the finite-difference error compensation. Each group in Figure 7 show:; in order, the reflector model, 3-D phase-shift modeling, migration without the compensation, and migration with the compensation. (a) Cube displays. 

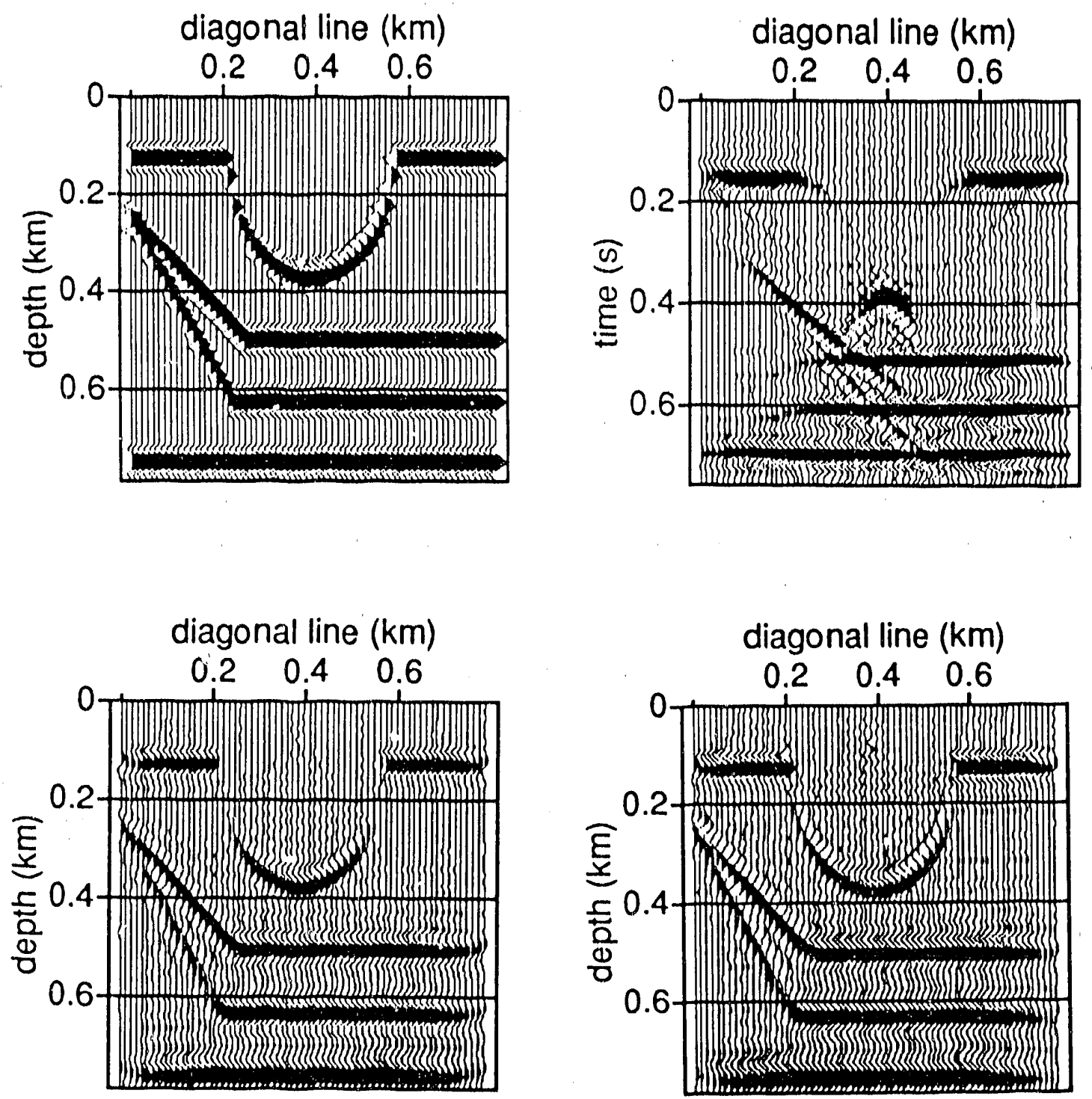

(b)

FIG. 7. (b) Vertical sections along the diagonal line $x=y$. 

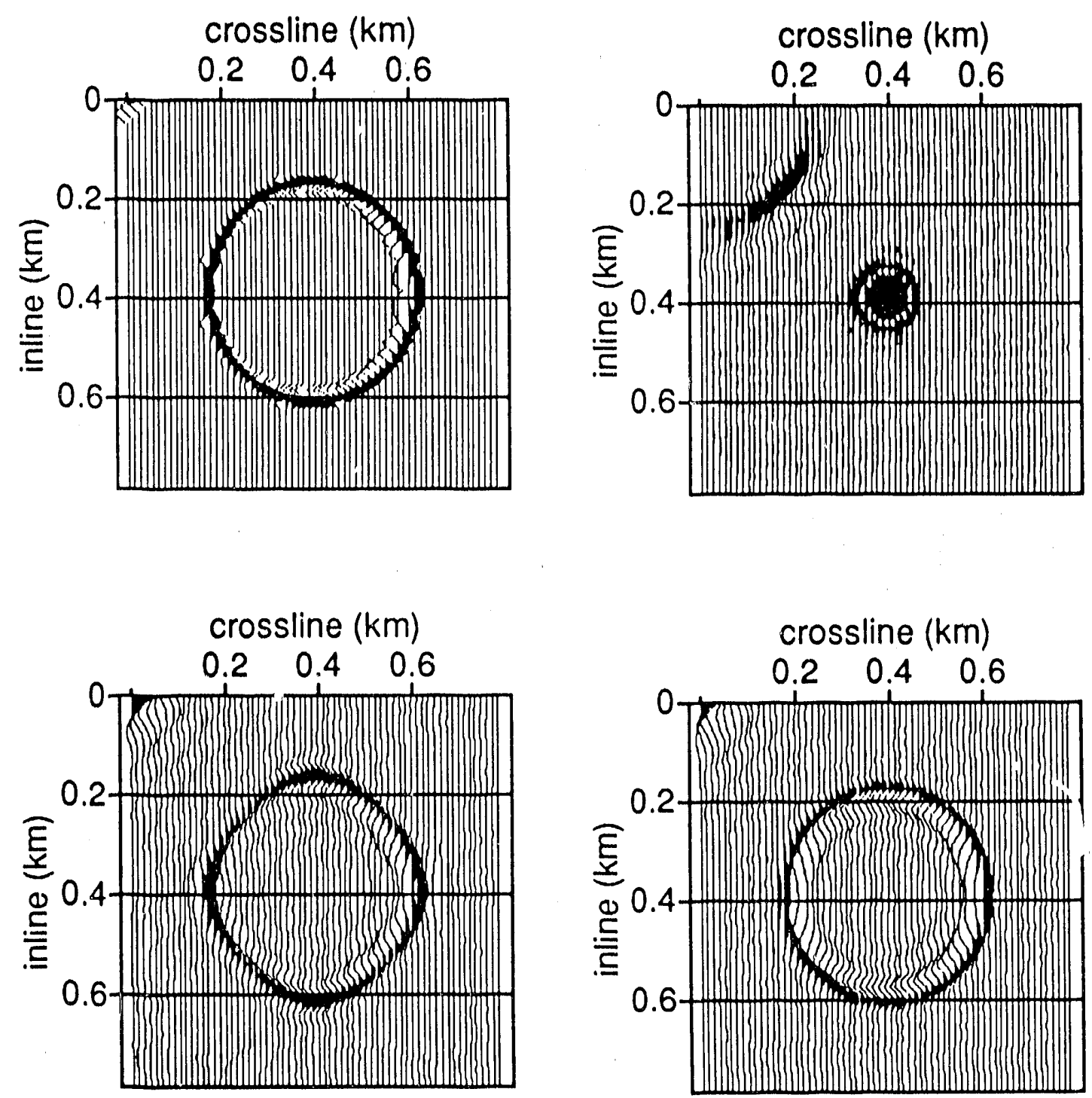

(c)

FIG. 7. (c) Depth slices at $z=.25 \mathrm{~km}$ and time slice at $t=0.40 \mathrm{~s}$. 

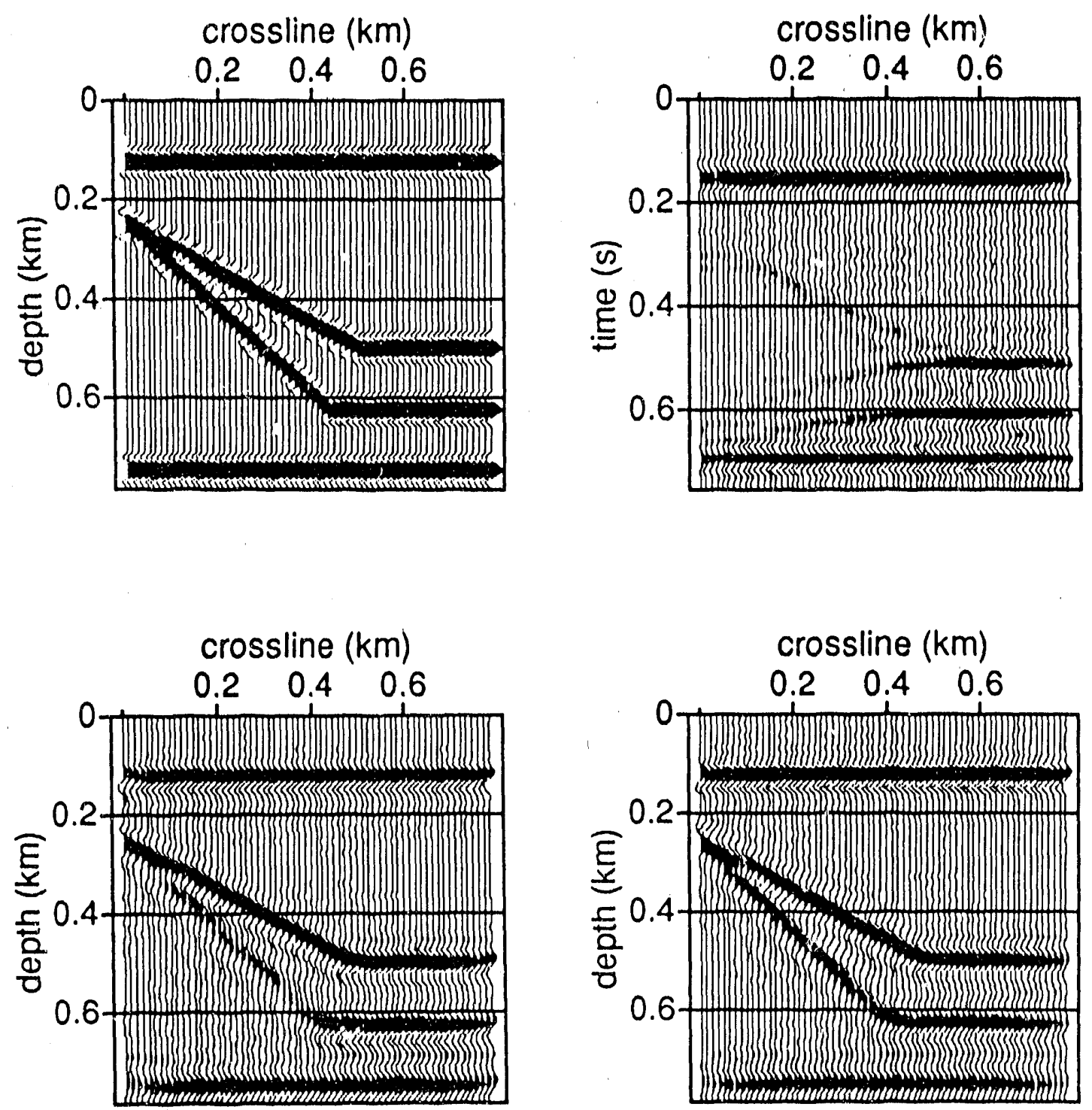

(d)

Fig. 7. (d) Vertical sections at $x=0$. 

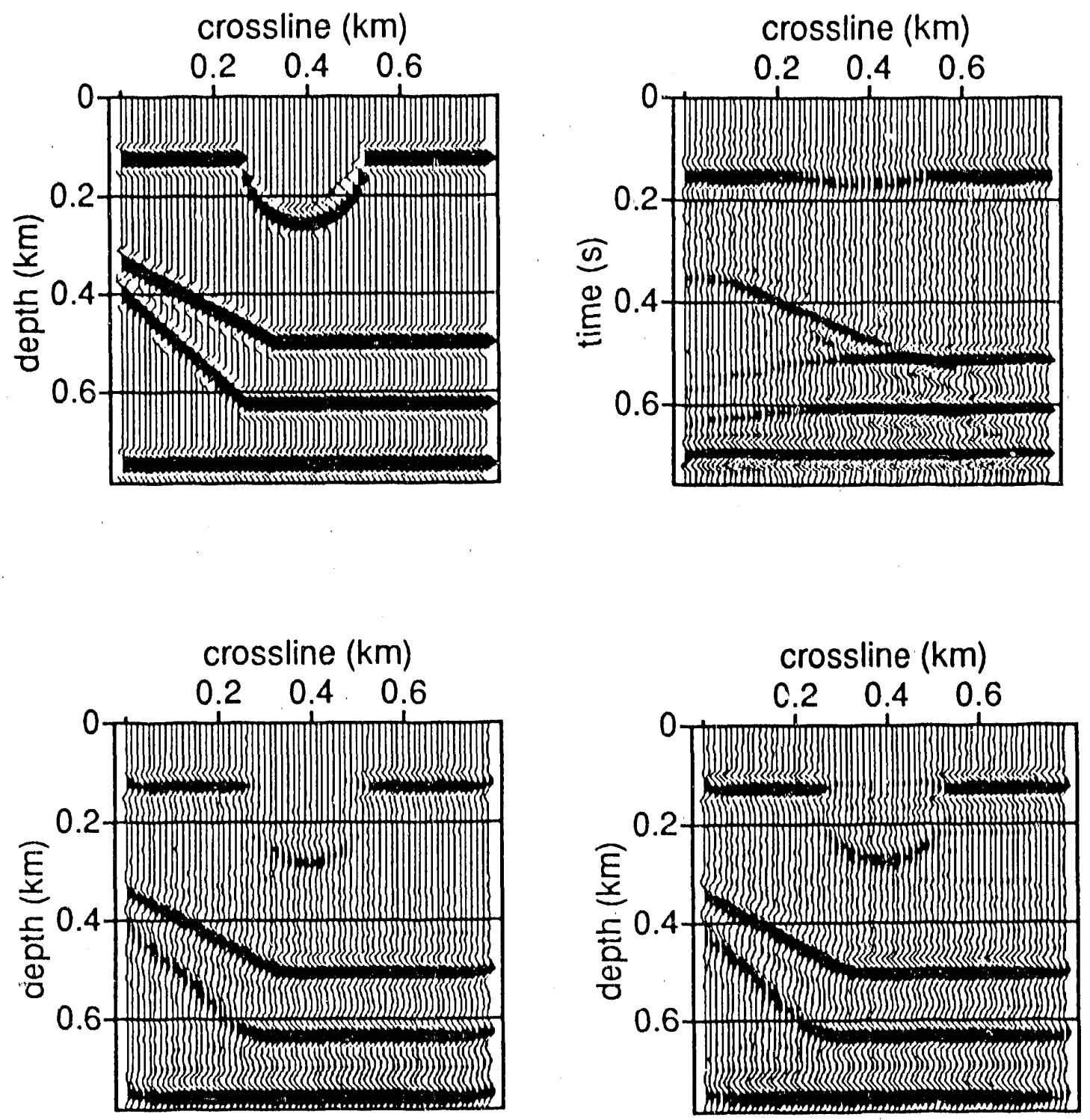

(e)

FIG. 7. (c) Vertical sections at $y=0.175 \mathrm{~km}$. 

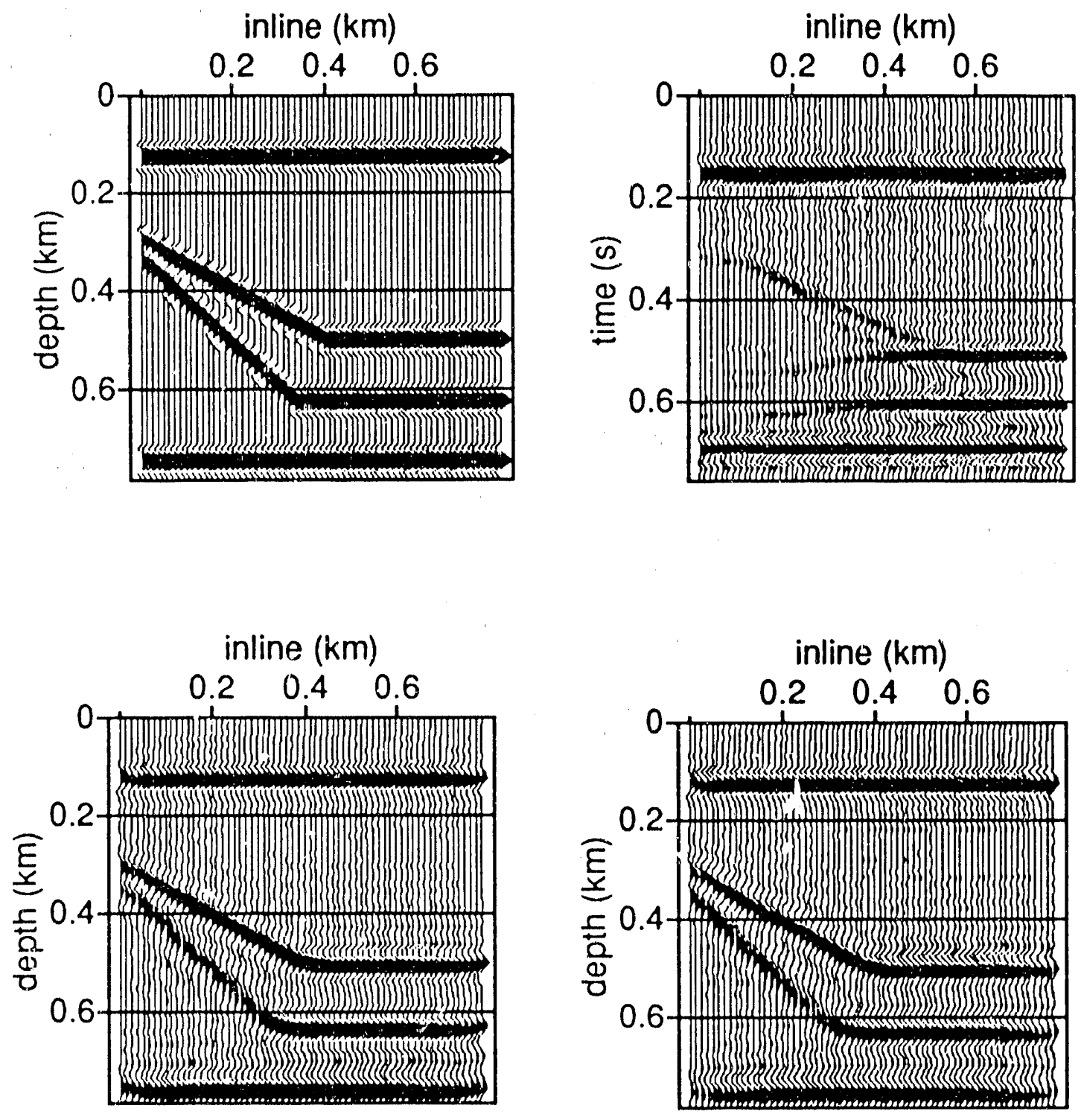

(f)

FIG. 7. (f) Vertical sections at $y=0.10 \mathrm{~km}$. 


\section{ACKNOWLEDGMEINTS}

This work benefits trom many discussions with Dave Hale and Ken Larner at Colorado School of Mines. Reviewing and suggested revisions of the paper by Dave Hale, Ken Larner, Jack Cohen and Norm Bleis'ein are greatly appreciated. Comments from Wendell Wiggins, Ron Chambers and Alfonso Gonzalez-Serrano at Western Geophysical were also helpful. Thanks for financial support are due to the sponsors of the Consortium Project on Seismic Inverse Methods for Complex Structures at the Center of Wave Phenomena of Colorado School of Mines and the United States Department of Energy, Grant Number DE-FG02-89ER14079. (This support does not constitute an endorsement by DOE of the views expressed in this paper.)

\section{REFERENCES}

Brown, D.L., 1983, Applications of operator separation in reflection seismology: Geophysics, 48, 288-294.

Claerbout, J.F., 1985, Imaging the earth's interior, Blackwell Scientific Publications, Inc.

Gazdag, J., 1978, Wave-equation migration with the phase-shift method: Geoplysics, $43,1342-1351$.

Gazdag, J., and Sguazzero, P., 1984, Migration of seismic data by phase-shift plus interpolation: Geophysics, 49, 124-131.

Graves, R.W., and Clayton, R.W., 1990, Modeling acoustic waves with paraxial extrapolators: Geophysics, 55, 306-319.

Hale, D., 1990, 3-D depth migration via McClellan transformations: Expanded Abstracts of 60th Annual International Meeting of Society of Exploration Geophysicists.

Kitchenside, P., 1988, Steep dip 3-D migration: some i.sues and examples: Expanded Abstracts of 58th Annual International Meeting of Society of Exploration Geophysicists, 976-978.

Kjartansson, E., 1979, Attenuation of seismic waves in rocks and applications in energy exploration: Ph.D. thesis, Stanford University.

Lee, M., and Suh, S., 1985, Optimization of one-way wave equation: Gcophysics, 50, $1634-1637$.

Ma, Z., 1982, Finite-difference migration with higher-order approximation: Oil Geophysical Prospecting, 17, 6-15.

Ristow, D., 1980, 3-D downward extrapolation of seismic data in particular by finite difference method: Ph.D. thesis, University of Utrecht, the Netherlands.

Rothman, D., Levin, S., and Rocca, F., 1985, Residual migration: Application and limitations: Geophysics, 50, 110-126.

Yilmaz, O., 1987, Seismic data processing, Society of Exploration Geophysicists, Tulsa, OK. 

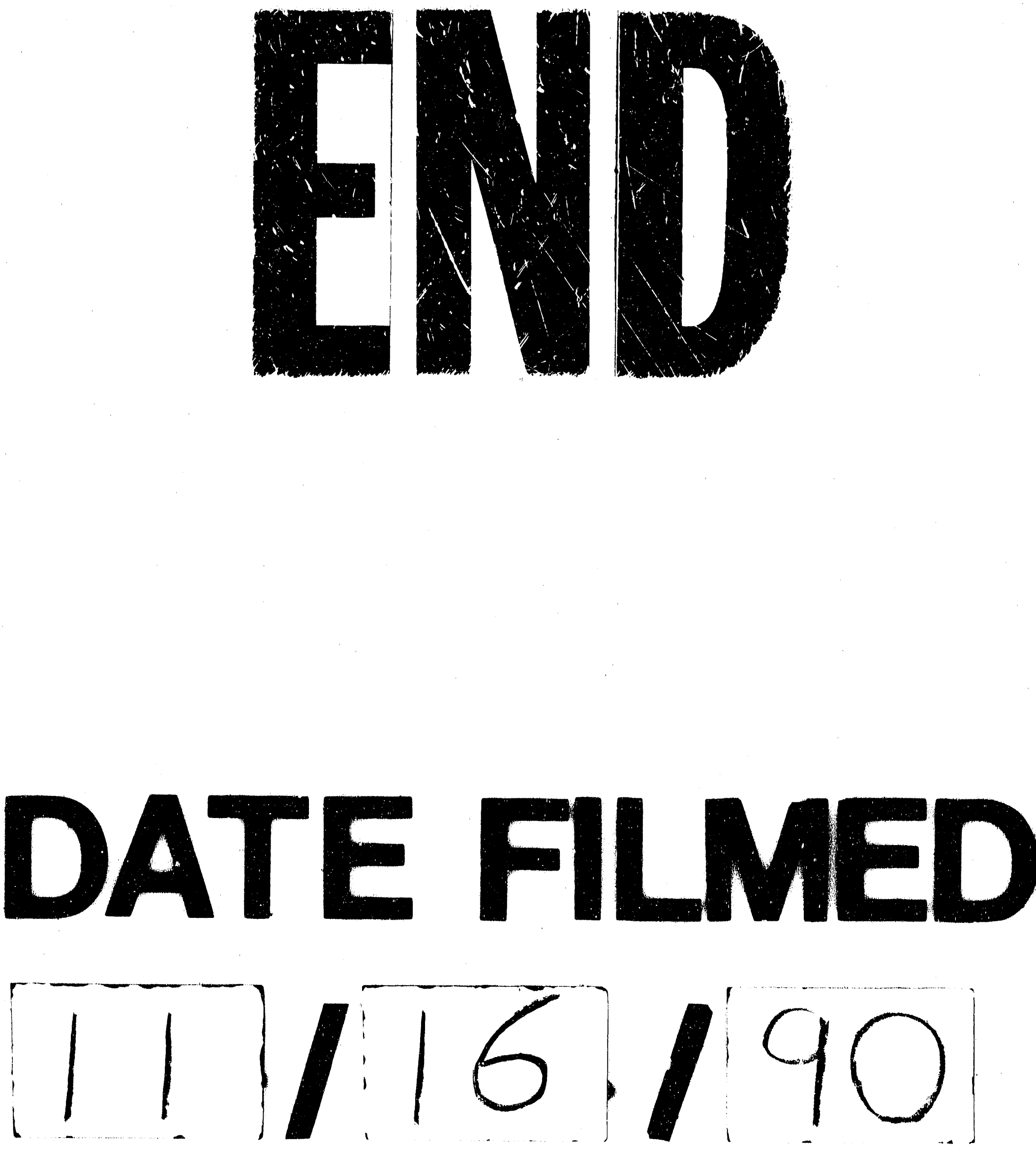
